\title{
Effect of supplementation of inulin in dietary on lactation performance, rumen fermentation, ruminal microbial profile and metabolites in dairy cows
}

\section{Yue Wang}

Chinese Academy of Agricultural Sciences Institute of Animal Science

\section{Xuemei Nan}

Chinese Academy of Agricultural Sciences Institute of Animal Science

\section{Yiguang Zhao}

Chinese Academy of Agricultural Sciences

\section{Linshu Jiang}

Beijing University of Agriculture

\section{Hui Wang}

Chinese Academy of Agricultural Sciences

\section{Dengke Hua}

Chinese Academy of Agricultural Sciences

\section{Fan Zhang}

Chinese Academy of Agricultural Sciences

\section{Yapin Wang}

Chinese Academy of Agricultural Sciences

Jun Liu

Langfang Academy of Agriculture and Forestry

Junhu Yao

Northwest A\&F University: Northwest Agriculture and Forestry University

Benhai Xiong ( $\square$ xiongbenhai@caas.cn )

State Key Laboratory of Animal Nutrition https://orcid.org/0000-0003-2773-5739

\section{Research}

Keywords: inulin, lactation performance, rumen fermentation, microbiota, metabolomics, dairy cow

Posted Date: December 18th, 2020

DOI: https://doi.org/10.21203/rs.3.rs-130241/v1 
License: (c) (i) This work is licensed under a Creative Commons Attribution 4.0 International License. Read Full License 


\section{Abstract}

\section{Background}

Inulin is a kind of fructo-oligosaccharide (FOS) derived mainly from Jerusalem artichoke and chicory tubers, which is also a soluble dietary fiber. Inulin has become a scientifically proven prebiotic product with satisfactory effects in improving the structure of intestinal flora, regulating blood lipid and glycemia, etc., in humans and monogastric. However, unlike monogastric animals, ruminal microbes are the largest microbiota in ruminants. The microflora profile and metabolism activity in the rumen are closely related to the health of dairy cows. This study investigated the effects of inulin on rumen fermentation parameters, ruminal microbiome and metabolites, as well as lactation performance and serum indexes in dairy cows. A total of 16 Holstein dairy cows with similar body condition were randomly divided into two groups ( $n=8$ per group), with inulin addition at 0 and $200 \mathrm{~g} / \mathrm{d}$ per cow, respectively. Experiment was lasted for 6 weeks including 1 week of adaptation period and 5 weeks of treatment period. At the end of the experimental period, the milk, serum and rumen fluid were sampled and analyzed. The rumen microbiota and metabolites were analyzed via 16S rRNA sequencing and untargeted metabolomics, respectively.

\section{Results}

The supplementation of inulin $(200 \mathrm{~g} / \mathrm{d}$ per cow) increased the milk yield $(P=0.001)$, milk protein $(P=$ 0.032), lactose rate $(P=0.004)$ and the proportion of saturated fatty acids (SFA) in milk $(P<0.001)$, while decreased the proportion of unsaturated fatty acids (USFA) $(P=0.041)$. Rumen $\mathrm{pH}(P=0.040)$ and concentration of $\mathrm{NH}_{3}-\mathrm{N}(P=0.024)$ were decreased, however, acetate $(P<0.001)$, propionate $(P=0.003)$, butyrate $(P<0.001)$ and lactic acid (LA) $(P=0.043)$ were increased. The total cholesterol (TC) $(P=0.008)$ and triglycerides (TG) $(P=0.01)$ in serum were also reduced. Additionally, the inulin addition elevated the relative abundance of several beneficial symbiotic and short-chain fatty acids (SCFA)-producing bacteria, such as Muribaculaceae (FDR-adjusted $P<0.01$ ), Acetitomaculum (FDR-adjusted $P=0.043$ ), and Butyrivibrio (FDR-adjusted $P=0.036$ ) etc., meanwhile elevated the levels of L-Lysine (FDR-adjusted $P=$ $4.24 \times 10^{-3}$ ), L-Proline (FDR-adjusted $P=0.0158$ ), L-Phenylalanine (FDR-adjusted $P=0.027$ ), etc. By contrast, several pathogens and ruminal bacteria abundant in high-fat diets, such as Escherichia-Shigella (FDR-adjusted $P=0.022$ ), Erysipelotrichaceae_UCG-004 (FDR-adjusted $P<0.01$ ) and RF39 (FDR-adjusted $P=0.042$ ) etc., were decreased along with the reduction of LysoPC (18:1(9Z)) (FDR-adjusted $P=1.03 \times$ $\left.10^{-3}\right)$, LysoPC (16:0) (FDR-adjusted $\left.P=0.0108\right)$, LysoPC $(18: 2(9 Z, 12 Z))$ (FDR-adjusted $P=1.65 \times 10^{-3}$ ) and 8-Methylnonenoate etc.

\section{Conclusion}


The results indicated the supplementation of inulin in dietary can increase the relative abundance of commensal microbiota and SCFAs-producing bacteria, meanwhile, upregulate amino acids (AAs) metabolism and downregulate lipid metabolism in rumen of dairy cows, which might further improve lactation performance and the level of serum lipids.

\section{Background}

Inulin is generally served as a reserve polysaccharide in plants. Statistically, it was contained in approximately 36,000 plants, of which Jerusalem artichoke (Helianthus tuberosus L.) tubers were the most abundant [1]. As a kind of fructo-oligosaccharide (FOS), inulin is mainly composed of $\beta$-Dfrutofuranose and glucopyranose residues connected by $\beta-(1,2)$ glucosidic bond [2]. There is a mild smell in inulin and with the characteristics of high sweetness and low calorific value. Although the inulin has high stability in water and tends to agglomerate when dispersed in water, [3] Ronkart et al. [4] reported that it could be slowly degraded into fructose and glucose in the solution with $\mathrm{pH}<4$ and the proper temperature for a period of time. Due to the existence of $\beta-(1,2)$ glycosidic bond structure, inulin cannot be digested and decomposed in the mouth, stomach and small intestine, and can only be partially fermented by Bifidobacteria in the colon. [2] Therefore, inulin is a scientifically proved and edible nondigestible polysaccharide that meets the definition of prebiotics. [2]

As a soluble dietary fiber, inulin has been widely studied in human and monogastric animals. Inulin can promote the growth of probiotics, such as Bifidobacteria and Lactobacilli, etc., and inhibit the proliferation of pathogenic and putrid bacteria, such as Clostridium pneumoniae, Enterococcus and Mould etc. [2], and meanwhile, the changes of intestinal flora caused by inulin fermentation can regulate the immune function of intestinal lymphoid tissue. [5] Chen et al. [6] found that inulin could delay the occurrence of immune diseases such as acute pancreatitis by regulating the homeostasis of the intestinal flora. On the other hand, inulin can induce the metabolic changes. It could promote the production of endogenous active substance cathelicidin related antimicrobial peptide (CRAMP). The effect depended on the production of short-chain fatty acids (SCFAs). In addition, a large number of SCFAs and lactic acid (LA) produced by inulin fermentation in the colon can not only stimulate intestinal peristalsis and prevent constipation, ${ }^{2}$ but also lower the $\mathrm{pH}$ value of the intestine and facilitate mineral absorption. [7] Additionally, inulin has been shown to have the ability to inhibit the degradation of ingested fat by lipolytic enzymes, thereby lowering blood lipids. [8] Also, the lipid-lowering effect may also be related to the regulation of cholesterol and triglycerides (TG) in blood by SCFAs produced by inulin fermentation. [9] In a word, inulin can improve the intestinal environment and body health in human and monogastric animals by changing the microbial population and metabolic processes.

However, studies on inulin in ruminants, especially in dairy cows, were quite limited. Previous studies supported that inulin could not exert prebiotic role in ruminants because the existence of the rumen, in which inulin would be strongly degraded. $[10,11]$ The fermentation process of inulin in the colon of monogastric animals is basically analogous to the process that occurs in the rumen of ruminants. [10] An in vitro rumen fermentation test in sheep showed that adding $1.5 \mathrm{~g} \mathrm{DM}$ inulin to an incubation bottles 
containing $1 \mathrm{~g}$ basic diet could significantly reduce the concentration of ammoniacal nitrogen $\left(\mathrm{NH}_{3}-\mathrm{N}\right)$, which showed the potential of inulin to improve rumen nitrogen utilization. [12] Zhao et al. [11] investigated the effects of inulin on rumen fermentation and microbial growth in goats by rumen simulation technology. The results showed that inulin treatment decreased the concentration of acetate, the ratio of acetate to propionate (A/P) and methane production, but increased the concentration of butyrate. Furthermore, the abundance of the Fibrobacter succinogenes and Ruminococcus flavefaciens were reduced, which indicated that inulin may inhibit the growth of partial rumen cellulose decomposition bacteria. Nevertheless, Umucalilar et al. [13] reported that inulin could only play a limited modifier role in regulating rumen fermentation parameters in vitro experiment. Another study on finishing beef showed that the supplementation of inulin in the diet diverted the rumen fermentation pattern from acetate to propionate and butyrate. Moreover, the a-diversity index of rumen bacteria was increased, in which the abundance of Bacteroides and Firmicutes were significantly increased, as well as weight and feed utilization [14]. Most of the previous studies on the effects of inulin on ruminants mainly focused on in vitro tests, which may restrict the knowledge and in-depth study of inulin's effects on the actual physiological regulation and production performance in ruminants.

Intestinal microbes are the most important microbial group in monogastric animals, and ruminant microbes have the same status in ruminants. The successful application of inulin in monogastric animals to improve the intestinal environment has aroused great interest in its potential roles on rumen function, and physiology and production performance on ruminants. Up to now, however, the effects of inulin on lactation performance, immune capacity, ruminal microorganism composition and metabolites activity in dairy cows have received few research attention. In this study, 16S rRNA sequencing and untargeted metabolomics technology were applied to investigate the effects of inulin on rumen microbial profile and the metabolites compositions, which was helpful to evaluate the feasibility of using inulin as feed additive in dairy cows.

\section{Material And Methods}

\section{Ethics statement}

The experimental animals, designs and operations in the present study were approved by the Animal Ethics Committee of the Chinese Academy of Agricultural Sciences (Beijing, China) (approval number: IAS-2019-9) and were in accordance with the recommendations of the academy's guidelines for animal research.

\section{Inulin}

The inulin (> 85\% purity, other sugars including fructose, sucrose and glucose $<15 \%$ ) added in this study was in powder form and extracted from Jerusalem artichoke (Helianthus tuberosus L.) tubers, which provided by Langfang Academy of Agriculture and Forestry (Hebei, China).

\section{Experimental Animals, Diets and Design}


The experiment was carried out on a well-managed and large-scale dairy farm in the suburbs of Beijing, China, which lasted 6 weeks composed by 1 week of adaptation and 5 week of treatments from midSeptember to late October. A total of 16 Holstein dairy cows with similar initial parity $(1.83 \pm 0.91)$, body weight (BW) (553 $\pm 17.3 \mathrm{~kg})$, days in milk (DIM) $(166 \pm 48.8 \mathrm{~d})$, milk yield $(33.7 \pm 2.64 \mathrm{~kg} / \mathrm{d})$ and milk somatic cell counts (SCC) (338 $\pm 64.5 \times 10^{3}$ cells $\left./ \mathrm{mL}\right)$ were randomly assigned to two groups, including control and inulin group $(n=8)$. The cows in the two groups were fed 3 times a day at 0700,1300 , and 1900h, respectively, with same total mixed rations (TMR; concentrate-to-forage ratio of 40:60). Cows were fed TMR and drink freely. The TMR ingredients and nutrient components are listed in Additional file 1: Table S1. Based on the basal diet, the inulin addition dose were 0 and $200 \mathrm{~g} / \mathrm{d}$ per cow in control and inulin group, respectively. The addition level of inulin in the current study was determined according to an independent dose screening test, which showed that $200 \mathrm{~g} / \mathrm{d}$ per cow had a significant effect on rumen fermentation and lactation performance in dairy cows (the data to be published). Therefore, this addition dose was select in the present study. The inulin was offered through oral dosing after the morning feeding.

\section{Milk Sample Collection and Body Weight Measurement}

The cows were milking 3 times a day at 0600, 1200 and 1800h, respectively, through automatic milking systems (Afimilk, Israel). At the last 3 consecutive days in the end phase of experiment, the milk samples were collected, the milk yield and BW were recorded. The BW was measured once a day on a floor scale before morning feeding. The average milk yield and BW in these 3 days were used as the representatives for the experiment period. The milk samples from the 3 times in a day were mixed at the ratio of 4:3:3 [15]. A part of the mixed milk samples were added with $0.6 \mathrm{mg} / \mathrm{mL}$ potassium dichromate as a preservative and stored at $4{ }^{\circ} \mathrm{C}$ for the analysis of milk composition. The other part of mixed milk samples were stored at $-20^{\circ} \mathrm{C}$ for the analysis of milk fatty acids (FAs).

\section{Milk Composition and Fatty Acids Analysis}

Milk protein, fat, lactose, urea nitrogen (MUN) and SCC were measured at the Beijing Dairy Cattle Center (Beijing, China) by Milk composition analyzer (Lactoscan SP, Funke Gerber, Berlin, Germany)

subsequently. The milk FAs was detected by acetyl chloride-methanolmethyl esterification method. The milk sample pretreatment referenced González-Córdova and Vallejo-Cordoba [16]. In addition, $4.5 \mathrm{~mL}$ of toluene was added to $0.5 \mathrm{~mL} F A$ triglycerides and mixed thoroughly as a standard measurement solution. The milk FAs composition were analyzed with gas chromatograph (Agilent $8860 \mathrm{GC}$, California, USA). The details were as follows: Column, dicyanopropyl polysiloxane column $(100 \mathrm{~m} \times 0.25 \mathrm{~mm}, 0.20 \mu \mathrm{m})$; The temperature of the column oven was $140^{\circ} \mathrm{C}$ for $5 \mathrm{~min}$, then was increased to $240^{\circ} \mathrm{C}$ at $4^{\circ} \mathrm{C} / \mathrm{min}$ for $5 \mathrm{~min}$; The injector and detector temperature were $260^{\circ} \mathrm{C}$ and $280^{\circ} \mathrm{C}$, respectively [16].

\section{Blood Sampling and Analysis}

Blood samples were collected through the caudal vein into procoagulant inert separation tubes (Saiao An Weiye Technology Development Co., Ltd., Beijing, China) at approximately $1 \mathrm{~h}$ before morning feeding on 
the last day of the experiment. . The blood samples were placed in a foam box with ice packs and centrifuged at $3000 \times \mathrm{g}$ for $15 \mathrm{~min}$ after brought back to the laboratory. The separated serum from each cow were collected and stored at $-20^{\circ} \mathrm{C}$ for the further analysis of biochemical indicators. The serum TG and total cholesterol (TC) concentration were analyzed by serum triglyceride determination kit (TR0100, Sigma-Aldrich, Missouri, USA) and cholesterol quantitation kit (MAK043, Sigma-Aldrich, Missouri, USA), respectively. The concentration of total protein (TP) was defined through biuret specific absorbance colorimetric method using total protein detection kit (Bai Olaibo technology Co., Ltd., Beijing, China). The serum albumin (ALB) concentration was detected by bromocresol green (BCG) method using BCG albumin assay kit (MAK124, Sigma-Aldrich, Missouri, USA). The concentration of serum globulin (GLO) was calculated by the difference between the concentration of TP and ALB. The blood urea nitrogen (BUN) concentration was determined by urea nitrogen kit (MS1812, Leigen Biotechnology Co., Ltd., Beijing, China).

\section{Rumen Fluid Sampling}

Rumen fluid samples were collected on the last day of this experiment. At $1 \mathrm{~h}$ before morning feeding, a gastric tube type rumen fluid sampler (MDW15, Colebo Equipment Co., Ltd., Wuhan, China) and a $200 \mathrm{~mL}$ syringe were used to collect rumen fluid sample from each cow. The first two tubes of rumen fluid were discarded to avoid saliva contamination, and $100 \mathrm{~mL}$ rumen fluid sample was collected from each cow [17]. The $\mathrm{pH}$ value of rumen fluid samples were immediately measured after collection by a portable $\mathrm{pH}$ meter (Bell Analytical Instruments Co., Ltd., Liaoning, China). Each rumen fluid sample was filtered through 4 layers of gauze and divided into six parts for the analysis of volatile fatty acids (VFAs), $\mathrm{NH}_{3}-\mathrm{N}$, rumen urea nitrogen (RUN), LA, ruminal bacteria, and metabolites, respectively.

\section{Rumen Fermentation IndicesMeasurement}

The concentration of VFAs were detected by gas chromatograph (Agilent 8890 GC, California, USA) [17]. The enzyme-labeled instrument (Multiskan ${ }^{\mathrm{TM}} \mathrm{FC}$, Thermo Fisher, Beijing, China) was used to measure $\mathrm{NH}_{3}-\mathrm{N}$ concentration [18]. The concentration of RUN and LA were detected by urea nitrogen kit (MS1812, Leigen Biotechnology Co., Ltd., Beijing, China) and LA detection kit (TC0733, Leigen Biotechnology Co., Ltd., Beijing, China), respectively. The ruminal microflora and metabolites were analyzed by $16 \mathrm{~S}$ rRNA sequencing and untargeted metabolomics techniques.

\section{Ruminal Microorganism DNA Extraction, PCR Amplification and Sequencing}

MagBeads Soil DNA Extraction Kit (SMDE-5010, Tiangen Biochemical Technology Co., Ltd., Beijing, China) was used to extract the total microbial DNA from 16 rumen fluid samples according to the manufacturer's instructions. DNA purity and concentration were detected with NanoDrop2000 (Thermo Fisher, New York, USA) and DNA integrity was assessed by $1 \%$ agarose gel electrophoresis. The $16 \mathrm{~S}$ V3 and V4 regions were amplified with forward primer 338F (5'-ACTCCTACGGGAGGCAGCAG-3') and reverse primer 806R (5'-GGACTACHVGGGTWTCTAAT-3'). The PCR reaction system $(20 \mu \mathrm{L})$ included $4 \mu \mathrm{L} 5 \times$ FastPfu Buffer, $2 \mu \mathrm{L} 2.5 \mathrm{mM}$ dNTPs, $0.8 \mu \mathrm{L}$ forward primer $(5 \mu \mathrm{M}), 0.8 \mu \mathrm{L}$ reverse primer $(5 \mu \mathrm{M}), 0.4 \mu \mathrm{L}$ 
FastPfu Polymerase, $0.2 \mu \mathrm{L}$ BSA, $10 \mathrm{ng}$ template DNA, and $\mathrm{dd}_{2} \mathrm{O}$. Three PCR replicates for each sample were mixed, and $5 \mu \mathrm{L}$ PCR products of each sample were detected by $2 \%$ agarose gel electrophoresis. The PCR products were purified using the AxyPrep DNA Gel Extraction Kit (AP-GX-250, Axygen Biosciences, Union City, USA) and were quantified with Quantus Fluorometer (E6150, Promega, Wisconsin, USA). The NEXTflex Rapid DNA-Seq Kit (5144-08, Bioo Scientific, Texas, USA) was used to construct the Miseq libraries. The Sequencing was conducted in the Miseq PE300 platform (Illumine, San Diego, USA) [17].

\section{Analysis of Sequencing Data}

The Trimmomatic software (version v 0.36 ) was used for quality control (QC) of raw sequences, and FLASH software (version 1.2.11, https://ccb.jhu.edu/software/F LASH/index.shtml) was used for splicing: i) The bases with tail mass value below 20 and the reads less than 50 bp length after QC were filtered, respectively, and the reads including $\mathrm{N}$ bases were removed; ii) According to overlap relation between PE reads, pairs of reads were merged into a sequence, with minimum overlap length of $10 \mathrm{bp}$; iii) The overlap area of mosaics sequence were allowed the maximum error ratio of 0.2 , the unmatched sequences were filtered out; iv) Samples were distinguished and the sequence direction was adjusted according to the barcode and primer at both ends of the sequences. The allowed mismatches of barcode and primer mismatch were 0 and 2, respectively. The UPARSE software (version 7.0.1090, http://drive5.com/uparse/) was used to perform the operational taxonomic units (OTU) sequence clustering, and select the sequences with more than $97 \%$ similar to the representative sequences of OTU, meanwhile, the chimeras were eliminated by UCHIME software (version 7.0, http://www. drive5.com/usearch/) and then the OTU table was generated. The ribosomal database project (RDP) classifier (http://rdp.cme.msu.edu/) was used to classify and annotate each sequence through comparing to Silva (Release132, http://www.arb-silva.de) with the comparison threshold of 70\% [19].

The alpha diversity analysis at OTU level was conducted by Mothur software (version 1.30.2, https://www.mothur.org /wiki/Download_mothur). The difference test of the alpha diversity index (Sobs, ACE, Chao, Shannon and Simpson) in the two groups were conducted by Wilcoxon rank-sum test. The abundance at various taxonomic levels and beta diversity distance were calculated through Qiime software (version 1.9.1, http://qiime.org/install/index.html). In the beta diversity analysis, the principal coordinates analysis (PCoA) and non-metric multidimensional scaling (NMDS) were analyzed at OTU level with the distance algorithm of weighted normalized Unifrac. The differential bacteria were analyzed through linear discriminant analysis effect size (LEfSe) software (http://huttenhower.sph.harvard.edu/galaxy/root?Tool_id=lefse_Upload). Specifically, non-parametric factorial Kruskal-Wallis (KW) sum-rank test was applied to detect the taxa with significantly differential abundance. The $P$-value from KW sum-rank test were adjusted by false discovery rate (FDR), with statistical significances declared at FDR-adjusted $P<0.05$. And then, linear discriminant analysis (LDA) was used to estimate the influence of abundance of each species on difference effect [20].

\section{LC-MS Metabolomics Analysis}


One hundred microliter thawed rumen fluid sample from each cow was transferred into a $1.5 \mathrm{~mL}$ centrifuge tube, and $400 \mu \mathrm{L}$ extraction solution (acetonitrile: methanol = 1: 1) was added and mixed thoroughly for $30 \mathrm{~s}$. After treated with low-temperature ultrasound for $30 \mathrm{~min}\left(5^{\circ} \mathrm{C}, 40 \mathrm{KHz}\right)$, the solution was placed at $4{ }^{\circ} \mathrm{C}$ for $30 \mathrm{~min}$ and centrifuged at $13,000 \times \mathrm{g}$ for $15 \mathrm{~min}$. The supernatant was removed and the precipitate was dried with nitrogen, and $120 \mu \mathrm{L}$ reconstituted solution (acetonitrile: water $=1: 1$ ) was added to reconstitute the remains. The solution was treated with low temperature ultrasonic for $5 \mathrm{~min}$ $\left(5^{\circ} \mathrm{C}, 40 \mathrm{KHz}\right)$ and centrifuged at $13,000 \times \mathrm{g}$ for 5 min again. The supernatant was pipetted into a sample injection vial with an inner cannula for LC-MS analysis [17]. The metabolites from all samples with equal volume were mixed to prepare a QC sample. Inserting a QC sample for every 10 samples to check the repeatability of the entire analysis process.

The LC-MS analysis was performed through the UPLC-Triple TOF system (Triple TOF5600, AB SCIEX, Massachusetts, USA). Ten microliter sample from each cow was detected through mass spectrometry after separated by BEH C18 chromatographic column (100 mm × $2.1 \mathrm{~mm}$ i.d., $1.8 \mu \mathrm{m})$ (Waters, Massachusetts, USA). The conditions of LC-MS were as follows: mobile phase A: water (containing $0.1 \%$ formic acid); mobile phase B: acetonitrile: isopropanol (1: 1) (containing $0.1 \%$ formic acid); Separation gradient of mobile phase (A: B): $80 \%$ : 20\% from 0 to $3 \mathrm{~min}, 5 \%$ : $95 \%$ from 3 to 9 min and maintained for 2 min, 95\%: 5\% from 13.0 to $13.1 \mathrm{~min}$ and maintained for $3 \mathrm{~min}$; The flow rate was $0.40 \mathrm{~mL} / \mathrm{min}$, and the column temperature was $40^{\circ} \mathrm{C}$. The signal acquisition of mass spectrum was adopted the positive and negative ion scanning mode with the scanning range of $50-1000 \mathrm{~m} / \mathrm{z}$. The ion spray voltage in positive and negative ion mode were $5,000 \mathrm{~V}$ and 4,000 V, respectively. The ion source heating temperature was $500^{\circ} \mathrm{C}$ with voltage of $20-60 \mathrm{~V}[21]$.

\section{Metabolomics Data Processing and Analysis}

The raw data was processed by Progenesis QI (Waters, Milford, USA) software for baseline filtering, peak identification, integration, retention time (RT) correction, peak alignment, and finally a data matrix including RT, mass-to-charge ratio (M/Z), and peak intensity was obtained. In order to reduce the error caused by sample preparation and instrument instability, the response intensity of the sample mass spectrum peak is normalized by the sum normalization method. Meanwhile, the variables in QC samples with the relative standard deviation (RSD) $>30 \%$ were deleted to obtain the final data matrix for subsequent analysis. The MS spectrometry information was matched with the human metabolome database (HMDB) (http://www.hmdb.ca/) and Metlin database (https://metlin.scripps.edu/) to obtain metabolites information [21].

The preprocessed data was uploaded to the Majorbio Cloud Platform (https://cloud.majorbio.com) for further analysis. The ropls package of R program (Version1.6.2) was used to perform principal component analysis (PCA) and orthogonal least partial squares discriminant analysis (OPLS-DA), which accompanied by 7 cycles of interactive verification to evaluate the stability of the model. In addition, Student's t-test (unpaired) was used for the determination of significant differences. The fold change (FC) was used to evaluate the change trend (up-regulation or down-regulation) of differential metabolites. The 
selection of significantly differential metabolites was based on the variable important in projection (VIP) obtained from the OPLS-DA model and the $P$-value from Student's t-test, which adjusted by FDR. The metabolites with VIP $>1$ and FDR-adjusted $P$-value $<0.05$ were identified as the significantly different metabolites between the two groups. The diagram of Venn was printed by Venn Diagram in R packages (version 1.6.20). The hierarchical clustering analysis (HCA) for significantly different metabolites was performed by Scipy package in Python software (version 1.0.0). The metabolite distance algorithm was bray Curtis. The hierarchical clustering method of metabolites was average. The metabolic pathways in which the differential metabolites clustered were annotated through the KEGG database (https://www.kegg.jp/kegg/pathway.html). The Scipy package in Python software (version 1.0.0) was conducted for pathway enrichment analysis [21]. The receiver operator characteristic (ROC) analysis was used to examine the metabolites that were critical to the intergroup differentiation by ROC ANALYSIS of SPSS Statistics (version 22, IBM, New York, USA). The closer the area under curve (AUC) value to 1, the higher the accuracy of the prediction was.

\section{Correlation Analysis}

The correlation analysis were conducted through the OmicShare tools (http://www.omicshare.com/tools). The correlations between differential bacteria and metabolites, rumen fermentation parameters and milk compositions, as well as significantly differential metabolites and milk compositions, were calculated by Spearman's correlation coefficient, respectively. The range of the correlation coefficient ( $r$ ) was from -1 to 1 . The $r>0$ and $<0$ were represented positive correlation and negative correlation, respectively. The $|r|$ value denoted the degree of correlation between variables. In particular, $r=-1,0$ and 1 were meant a completely negative correlation, uncorrelated and a completely positive correlation, respectively. Correlation significance $P$-value below 0.05 and 0.01 were served as significant and extremely significant correlation, respectively.

\section{Statistical Analysis}

Data statistics was performed by SPSS Statistics (version 22, IBM, New York, USA). The data of body condition information in dairy cows (parity, BW, DIM, and SCC), milk yield and compositions, milk fatty acids compositions, rumen fermentation parameters, and alpha diversity indices were analyzed through one-way ANOVA and Student's t-test, with statistical significances declared at $P<0.05$, a tendency was declared at $0.05<P<0.10$.

\section{Results}

\section{Effect of Inulin Supplementation on Lactation Performance}

The milk yield and compositions are showed in Table 1. With the supplementation of inulin, the dry matter intake (DMI) $(P=0.003)$, milk yield $(P=0.001)$, energy corrected milk $(\mathrm{ECM})$, fat corrected milk (FCM) $(P<0.001)$, milk protein $(P=0.042)$ and lactose $(P=0.004)$ were significantly increased, while milk fat was showed a tendency of increase $(P=0.075)$. Whereas, the MUN $(P=0.023), \operatorname{SCC}(P=0.036)$ and 
the fat to protein ratio $(F / P)$ was significantly decreased $(P=0.027)$, but the $F / P$ was still in the normal rage (1.12-1.36).

The effect of adding inulin on the level of milk FAs is listed in Table 2. Compared with control group, the proportion of saturated fatty acid (SFA) in inulin group was significantly increased $(P<0.001)$, mainly including the significant increase of $\mathrm{C} 6: 0(P=0.034), \mathrm{C} 8: 0(P=0.006), \mathrm{C} 10: 0(P=0.038), \mathrm{C} 12: 0(P=$ $0.001)$ and $\mathrm{C} 16: 0(P=0.001)$. While, the proportion of $\mathrm{C} 18: 0(P=0.002)$ and $\mathrm{C} 22: 0(P=0.031)$ were significantly reduced. On the other hand, the proportion of unsaturated fatty acid (UFA) in inulin group was significantly declined $(P=0.041)$, which were mainly attributed to the decrease of polyunsaturated fatty acids (PUFA) $(P=0.013)$. Among them, the proportion of C18:2 cis-6 $(P=0.011)$ and $\mathrm{C} 18: 3 \mathrm{n} 3(P<$ $0.001)$ were significantly reduced. Additionally, the level of short and medium-chain fatty acids (SMCFA) in inulin group was significant increase $(P<0.001)$.

\section{Effect of Inulin Supplementation on Serum Indexes}

As shown in Table 3, compared with control group, the concentration of TC $(P=0.008)$ and TG $(P=0.01)$ were significantly declined in the inulin group, while the level of TP, ALB, GLO and BUN had no significant difference $(P>0.05)$.

\section{Effects of Inulin Supplementation on Rumen Fermentation Characteristics}

The rumen fermentation parameters were listed in Table 4. The $\mathrm{pH}$ value in rumen was significantly declined $(P=0.040)$ with the addition of inulin, which accompanied by the significant increase of the concentration of acetate $(P<0.001)$, propionate $(P=0.003)$, butyrate $(P<0.001)$, isobutyrate $(P=0.002)$, valetate $(P=0.001)$, isovaletate $(P<0.001)$ and LA $(P=0.043)$. Meanwhile, the significant decrease of the concentration of $\mathrm{NH}_{3}-\mathrm{N}(P=0.024)$ was also observed in inulin group.

\section{Effect of Dietary Supplementation of Inulin on the Richness, Diversity and Composition of Ruminal Bacteria}

A total of 1,349,120 effective $16 \mathrm{~S}$ rRNA sequences were detected in 16 rumen fluid samples and 2,337 OTUs were obtained by performing OTU clustering on non-repetitive sequences according to $97 \%$ similarity. Rarefaction curves (Additional file 1:Figure S1) showed that the current sequencing depth and sample size were sufficient to assess the microbial diversity, total species richness and core species number of rumen fluid samples. The a-diversity analysis revealed that the ACE $(P=0.031)$, Chao $(P=$ $0.017)$ and Shannon $(P=0.026)$ indexes in inulin group were significantly increased, the Simpson index also showed a tendency of rise $(P=0.071)$, which illustrated that the addition of inulin increased the ruminal microbial community richness and diversity (Table 5).

The RDP classifier Bayesian algorithm was used to perform taxonomic analysis on the OTU representative sequences, and 21 bacteria phyla and 304 genera were obtained from 16 samples (Additional file 1:Table S2 and S3). At the phylum level, Bacteroidota (48.4 $\pm 0.47 \%$ and $51.7 \pm 0.36 \%$ ), 
Firmicutes ( $46.3 \pm 0.41 \%$ and $42.3 \pm 0.38 \%)$, Patescibacteria $(1.81 \pm 0.13 \%$ and $1.85 \pm 0.11 \%)$ and Actinobacteriota $(1.43 \pm 0.25 \%$ and $1.82 \pm 0.17 \%)$ were the dominant bacteria in control and inulin groups, respectively (Figure 1A and Additional file 1: Figure S2 A and B). At the genus level, Prevotella (36.8 $\pm 0.41 \%$ and $39.5 \pm 0.37 \%)$, Oscillospirales_NK4A214_group $(4.70 \pm 0.11 \%$ and $5.39 \pm 0.24 \%)$, Succiniclasticum (3.89 $\pm 0.24 \%$ and $3.78 \pm 0.20 \%)$, Ruminococcus $(4.15 \pm 0.32 \%$ and $3.90 \pm 0.17 \%$ ) and Muribaculaceae $(2.18 \pm 0.10 \%$ and $5.59 \pm 0.15 \%)$ were the predominant genera in control and inulin groups, respectively (Figure 1B and Additional file 1:Figure S2 C and D).

\section{Significantly Different Ruminal Bacteria between the Control and Inulin Groups}

The $\beta$-diversity analysis was performed to explore differences in rumen microbial community between the two groups (Figure 2). The PCoA and NMDS plots based on the Bray-Curtis distance matrix showed that the points representing rumen microorganisms in control and inulin groups respectively were significantly separated in different quadrants on the coordinate axis, which indicated inulin intake had a distinct effects on rumen microbial species and abundance. Venn diagram performed on the samples of OTU with $97 \%$ identity identified 87 and 117 unique OTU in control and inulin groups, respectively (Additional file 1: Figure S3). Further, the significantly different abundant ruminal bacteria between the two groups were identified through LEfSe analysis and LDA (Figure 3 A and B). The relative abundance of Muribaculaceae (FDR-adjusted $P<0.01$ ), Butyrivibrio (FDR-adjusted $P=0.036$ ), Prevotellaceae_NK3B31_group (FDR-adjusted $P=0.032)$, Eubacterium_coprostanoligenes_group $(P=$ 0.043), Lachnospiraceae_XPB1014_group (FDR-adjusted $P=0.035$ ), Acetitomaculum (FDR-adjusted $P=$ 0.043), Eubacterium_hallii_group (FDR-adjusted $P=0.031$ ), Lachnospiraceae (FDR-adjusted $P=0.026$ ) and Veillonellaceae_UCG-001 (FDR-adjusted $P=0.036$ ) were significantly increased in inulin groups compared with control group. In contrast, Erysipelotrichaceae_UCG-004 (FDR-adjusted $P<0.01$ ), Erysipelotrichaceae_UCG-008 (FDR-adjusted $P<0.01$ ), Clostridia_UCG-014 (FDR-adjusted $P=0.031$ ), Selenomonas (FDR-adjusted $P=0.040$ ), Bacteroidales_BS11_gut_group (FDR-adjusted $P=0.037$ ), Escherichia-Shigella (FDR-adjusted $P=0.022$ ), Anaerobiospirillum (FDR-adjusted $P=0.041$ ), Succinivibrionaceae_UCG_001 (FDR-adjusted $P=0.042$ ), Syntrophococcus (FDR-adjusted $P=0.037$ ) and $R F 39$ (FDR-adjusted $P=0.042$ ) were significantly decreased in rumen with the inulin addition (Additional file 1:Table S3).

\section{Effect of Dietary Supplementation of Inulin on Ruminal Metabolites}

The rumen metabolites were analyzed through untargeted metabolomics techniques. The total ion chromatograms (TIC) plot of QC samples in positive and negative ion mode are shown in Additional file 1: Figure S4 A and B. The overlap of QC samples revealed the well repeatability and high-accuracy of the data. The unsupervised multivariate statistical analysis, PCA, generally reflected that a distinct difference existed in the ruminal metabolites between the two groups and a less degree of variation among samples within a group in positive and negative ion modes (Additional file 1:Figure S5 A and B). Further OPLS-DA provided a supervised discriminant analysis method, which could further distinguish the differences of ruminal metabolites between control and inulin groups and improve the effectiveness and analytical 
capabilities of the model (Figure 4). In OPLS-DA plots, $R^{2} Y$ and $Q^{2}$ were used to evaluate the modeling and prediction ability of OPLS-DA model respectively. The cumulative value of $R^{2} Y$ and $Q^{2}$ in positive ( 0.995 and 0.806 ) and negative ( 0.907 and 0.839 ) ion model were all above 0.80 , which illustrated the stability and reliability of the model (Figure 4 A and C). Response permutation testing (RPT) was a randomized sequencing method to evaluate the accuracy of OPLS-DA models. As shown in Figure 4 B and $D$, the value of $R^{2}(0.953$ and 0.931$)$ and $Q^{2}(-0.0067$ and $-0.082<0)$ in positive and negative ion models revealed a well accuracy of OPLS-DA models.

\section{Significantly Different Ruminal Metabolites between the Control and Inulin Groups}

A total of 99 differential metabolites in rumen (64 in positive and 35 in negative ion models) between the control and inulin groups were detected with VIP $>1$ and FDR-adjusted $P<0.05$ (Additional file 1: Table S4). Among them, lipids and lipid-like molecules, organic acids and derivatives, organic oxygen compounds and organoheterocyclic compounds were accounted for $37.0 \pm 0.48 \%, 22.2 \pm 0.32 \%, 16.7 \pm$ $0.11 \%$ and $14.8 \pm 0.24 \%$, respectively (Additional file 1:Figure S6). The top 70 ruminal metabolites were selected for HCA analysis (Additional file 1: Figure S7). The differential metabolites between the two groups were divided into two cluster. The relative expression of PC (16:0/0:0), LysoPC (16:0), palmitoyl glucuronide, R-palmitoyl-(2-methyl) ethanolamide, 2-0-Protocatechuoylalphitolic acid, sphinganine, LysoPC (18:2(9Z, 12Z)) and LysoPC (18:1(9Z)) etc. were higher in control group than in inulin group. While, the relative expression of deltonin, 3,4,5-trihydroxy-6-(2-oxoethoxy) oxane-2-carboxylic acid, LProline, 2-Hydroxycinnamic acid, L-Tyrosine, L-Phenylalanine, L-Tyrosine, daidzein and L-Lysine etc., were elevated in inulin group compared with control group.

Furthermore, several significantly differential ruminal metabolites with VIP $\geq 2$ and FDR-adjusted $P<0.05$ between the two groups were screened out. Compared with control group, LysoPC (18:1(9Z)) (FDRadjusted $\left.P=1.03 \times 10^{-3}\right)$, LysoPC (16:0) (FDR-adjusted $\left.P=0.0108\right)$, LysoPC $(18: 2(9 Z, 12 Z)$ ) (FDR-adjusted $P=1.65 \times 10^{-3}$ ), Phenylmethylglycidic ester (FDR-adjusted $P=1.19 \times 10^{-3}$ ), N-Acetylcadaverine (FDRadjusted $P=0.0353$ ) and 8-Methylnonenoate (FDR-adjusted $P=1.60 \times 10^{-3}$ ) were significantly decreased in the inulin group. However, the L-Lysine (FDR-adjusted $P=4.24 \times 10^{-3}$ ), L-Proline (FDR-adjusted $P=$ 0.0158), L-Phenylalanine (FDR-adjusted $P=0.027$ ), daidzein (FDR-adjusted $P=0.0130$ ), uracil (FDRadjusted $P=0.0396$ ), deltonin (FDR-adjusted $P=0.0393$ ) and L-Tyrosine (FDR-adjusted $P=0.0353$ ) were significantly increased in the rumen with the supplementation of inulin (Additional file 1: Figure $\mathbf{S 8}$ and Table S4).

Receiver operator characteristic curve was performed to evaluate whether the significantly differential metabolites screened were critical to the intergroup differentiation. As shown in Figure 5, the AUC of 8Methylnonenoate, LysoPC (18:1(9Z)), LysoPC (18:2(9Z, 12Z)), L-Lysine, LysoPC (16:0) and L-Proline were all above 0.95 , which meant these significantly differential ruminal metabolites were representative to illustrate the effect of inulin on the shift of rumen metabolism.

\section{Metabolic pathway enrichment analysis of differentially abundant metabolites}

Page 13/37 
KEGG pathway enrichment analysis showed that the supplementation of inulin mainly affected the lipid and amino acid metabolism, vitamin metabolism, biosynthesis of plant secondary metabolites and protein metabolism in the rumen of dairy cows (Table 6).

\section{Correlation analysis among differential ruminal bacteria, metabolites, lactation and rumen fermentation performance.}

Spearman correlation coefficient was used to calculate the correlation among several indicators (Figure 6). Correlation analysis between significantly differential bacteria and milk components showed that the milk yield was positively associated with Muribaculaceae $(r=0.521$, FDR-adjusted $P=0.042)$, Butyrivibrio $(r=0.602$, FDR-adjusted $P=0.028)$ and Prevotellaceae_NK3B31_group $(r=0.607$, FDR-adjusted $P=$ 0.027), while negatively associated with Escherichia-Shigella $(r=-0.613$, FDR-adjusted $P=0.007)$. Milk protein was positively associated with Muribaculaceae ( $\mathrm{r}=0.595$, FDR-adjusted $P=0.015)$, Eubacterium_hallii_group $(\mathrm{r}=0.569$, FDR-adjusted $P=0.037)$ and Prevotellaceae_NK3B31_group $(\mathrm{r}=$ 0.579 , FDR-adjusted $P=0.039)$, but negatively associated with Erysipelotrichaceae_UCG_008 $(r=0.488$, FDR-adjusted $P=0.044)$ and Erysipelotrichaceae_UCG_O04 ( $r=0.510$, FDR-adjusted $P=0.044)$. The lactose was positively associated with Muribaculaceae $(r=0.585$, FDR-adjusted $P=0.025)$, Eubacterium_coprostanoligenes_group $(r=0.565$, FDR-adjusted $P=0.047)$, Eubacterium_hallii_group $(r=$ 0.602, FDR-adjusted $P=0.023)$, Treponema $(r=0.573$, FDR-adjusted $P=0.037)$ and Saccharofermentans ( $\mathrm{r}=0.582$, FDR-adjusted $P=0.034)$, but negatively associated with Escherichia-Shigella $(\mathrm{r}=-0.481$, FDRadjusted $P=0.048$ ). Milk fat was positively associated with Muribaculaceae $(\mathrm{r}=0.543$, FDR-adjusted $P=$ $0.041)$, Butyrivibrio $(r=0.476$, FDR-adjusted $P=0.042)$, Prevotellaceae_NK3B31_group $(r=0.468$, FDRadjusted $P=0.046)$ and Acetitomaculum ( $r=0.584$, FDR-adjusted $P=0.038)$. Milk SCC was negatively associated with Muribaculaceae $(\mathrm{r}=-0.759$, FDR-adjusted $P=0.011)$, Butyrivibrio $(\mathrm{r}=-0.678$, FDRadjusted $P=0.014)$, Eubacterium_hallii_group and Eubacterium_coprostanoligenes_group $(r=-0.743$, FDR-adjusted $P=0.010)$, while positively associated with Erysipelotrichaceae_UCG_008 $(r=0.575$, FDRadjusted $P=0.031)$, Escherichia-Shigella $(r=0.594$, FDR-adjusted $P=0.022)$ and Anaerobiospirillum $(r=$ 0.594, FDR-adjusted $P=0.031$ ) (Figure 6A).

In addition, the correlation analysis between significantly differential bacteria and rumen fermentation parameters revealed that, acetate was positively associated with Muribaculaceaec(r $=0.712$, FDRadjusted $P=0.002)$, Eubacterium_coprostanoligenes_group $(r=0.629$, FDR-adjusted $P=0.014)$ and Acetitomaculum $(r=0.700$, FDR-adjusted $P=0.003)$. Propionate was positively associated with Muribaculaceae $(r=0.649$, FDR-adjusted $P=0.007)$, Eubacterium_coprostanoligenes_group $(r=0.622$, FDR-adjusted $P=0.010)$, Eubacterium_hallii_group $(\mathrm{r}=0.670$, FDR-adjusted $P=0.021)$, Treponema $(\mathrm{r}=$ 0.608 , FDR-adjusted $P=0.023)$ and Saccharofermentans $(r=0.626$, FDR-adjusted $P=0.031)$. Butyrate was positively associated with Muribaculaceae $(r=0.739$, FDR-adjusted $P=0.001)$, Eubacterium_coprostanoligenes_group $(r=0.719$, FDR-adjusted $P=0.002)$ and Butyrivibrio $(r=0.700$, FDR-adjusted $P=0.003)$, while negatively associated with Escherichia-Shigella $(r=-0.681$, FDR-adjusted $P=0.032)$. Prevotellaceae_NK3B31_group was positively associated with isovaletate $(r=0.644$, FDRadjusted $P=0.030)$ and isobutyrate $(r=0.602$, FDR-adjusted $P=0.041)$. The LA was positively 
associated with Butyrivibrio ( $\mathrm{r}=0.639$, FDR-adjusted $P=0.034)$, Prevotellaceae_NK3B31_group $(\mathrm{r}=0.799$, FDR-adjusted $P<0.001)$, Acetitomaculum ( $\mathrm{r}=0.678$, FDR-adjusted $P=0.019)$ and

Eubacterium_hallii_group $(\mathrm{r}=0.653$, FDR-adjusted $P=0.017)$. However, the $\mathrm{NH}_{3}-\mathrm{N}$ was negatively associated with Muribaculaceae $(\mathrm{r}=-0.735$, FDR-adjusted $P=0.001)$, Eubacterium_coprostanoligenes_group $(r=-0.685$, FDR-adjusted $P=0.030)$, Butyrivibrio $(r=-0.648$, FDRadjusted $P=0.028)$, Eubacterium_hallii_group $(\mathrm{r}=-0.650$, FDR-adjusted $P=0.036)$ and Treponema $(\mathrm{r}=$ -0.653 , FDR-adjusted $P=0.021$ ) (Figure 6B).

The correlation between significantly differential metabolites and microbacteria is showed in Figure 6C. LysoPC (16:0), LysoPC (18:2(9Z, 12Z)) and LysoPC (18:1(9Z)) were negatively associated with Muribaculaceae $(\mathrm{r}=-0.806$, FDR-adjusted $P<0.001 ; \mathrm{r}=-0.740$, FDR-adjusted $P=0.001 ; \mathrm{r}=-0.717$, FDRadjusted $P=0.022)$ and Prevotellaceae_NK3B31_group $(r=-0.730$, FDR-adjusted $P=0.043 ; r=-0.676$, FDR-adjusted $P=0.024 ; \mathrm{r}=-0.649$, FDR-adjusted $P=0.031)$, but positive associated with EscherichiaShigella $(r=-0.643$, FDR-adjusted $P=0.030 ; r=-0.713$, FDR-adjusted $P=0.024 ; r=-0.717$, FDR-adjusted $P$ $=0.036)$ and Anaerobiospirillu $(\mathrm{r}=-0.735$, FDR-adjusted $P=0.038 ; \mathrm{r}=-0.675$, FDR-adjusted $P=0.042 ; \mathrm{r}=$ -0.694, FDR-adjusted $P=0.043$ ). N-Acetylcadaverine were positively correlated with Escherichia-Shigella ( $\mathrm{r}$ $=-0.572$, FDR-adjusted $P=0.042)$, Erysipelotrichaceae_UCG_008 $(r=-0.566$, FDR-adjusted $P=0.045)$ and Erysipelotrichaceae_UCG-004 $(r=-0.562$, FDR-adjusted $P=0.046)$. Uracil was positively correlated to Eubacterium_hallii_group ( $r=0.673$, FDR-adjusted $P=0.025)$. L-Lysine, L-Proline and L-Tyrosine were positively associated with Muribaculaceae $(r=0.602$, FDR-adjusted $P=0.031 ; r=0.585$, FDR-adjusted $P=$ $0.035 ; \mathrm{r}=0.607$, FDR-adjusted $P=0.032)$, Eubacterium_hallii_group $(\mathrm{r}=0.624$, FDR-adjusted $P=0.042 ; \mathrm{r}=$ 0.580 , FDR-adjusted $P=0.024 ; \mathrm{r}=0.613$, FDR-adjusted $P=0.031)$ and Prevotellaceae_NK3B31_group $(\mathrm{r}=$ 0.705 , FDR-adjusted $P=0.032 ; \mathrm{r}=0.584$, FDR-adjusted $P=0.047 ; \mathrm{r}=0.631$, FDR-adjusted $P=0.040$ ).

As shown in Figure 6D, the relevance between significantly differential metabolites and milk compositions showed that, the milk SCC was positively associated with LysoPC (16:0) $(r=0.772$, FDRadjusted $P=0.021)$, LysoPC $(18: 2(9 Z, 12 Z))(r=0.752$, FDR-adjusted $P=0.035)$, LysoPC $(18: 1(9 Z))(r=$ 0.798 , FDR-adjusted $P=0.023$ ) and N-Acetylcadaverine ( $r=0.683$, FDR-adjusted $P=0.041$, while negatively associated with L-Tyrosine $(r=-0.677$, FDR-adjusted $P=0.033)$ and L-Lysine $(r=-0.680$, FDRadjusted $P=0.037)$. Milk fat and protein was positively associated with L-Proline ( $\mathrm{r}=-0.741$, FDRadjusted $P=0.040 ; \mathrm{r}=-0.769$, FDR-adjusted $P=0.030)$ and L-Lysine $(\mathrm{r}=-0.785$, FDR-adjusted $P=0.036 ; \mathrm{r}$ $=-0.744$, FDR-adjusted $P=0.040$ ). Additionally, milk protein was also positively associated with LTyrosine $(r=-0.750$, FDR-adjusted $P=0.035)$. Moreover, uracil was positively related with lactose $(r=$ 0.679 , FDR-adjusted $P=0.045$ ).

\section{Discussion}

Even the encouraging results of effects of inulin on human and monogastric animal health especially the gut flora ecology have motivated researchers to explore its potentiality on ruminants like dairy cattle, beef, goat and sheep etc. [10-14], limited information was available for the effect of inulin in ruminants presently, and no consensus has been reached yet [22]. In the present study, the increase of milk yield 
after inulin addition in dietary might be attributed to the sweetness of inulin, ${ }^{2}$ which could increase its palatability and DMI. Moreover, as a nonstructural carbohydrate, the intake of inulin significantly increased the concentration of VFAs in the rumen, which provided sufficient energy for lactation [22]. In the current study, the increase in milk protein ratio might be due to the following two reasons: i) the inulin addition provided energy substrate, which reduced the amount of AAs used for energy supply, and thus increased microbial protein (MCP) synthesis in rumen, along with the decreased $M U N$ and $\mathrm{NH}_{3}-\mathrm{N}$ concentration observed in this study, and the nitrogen utilization by rumen microorganisms was enhanced. Besides, the increase of MCP entering the small intestine promoted the synthesis of milk proteins in the mammary gland [23]; ii) the increase of propionate concentration in the rumen after the inulin supplementation could stimulate insulin secretion, which increased the absorption of AAs by the mammary glands, thereby increasing the milk protein percentage $[22,23]$. The increase of AAs levels were observed in the results of metabolomics in our study. The uptake of glucose by the mammary glands directly affects the milk lactose content. After inulin supplementation, the increased precursor of gluconeogenesis in rumen, such as propionate, LA and AAs, were absorbed into the blood through the rumen wall, which may promote more glucose from the blood were obtained by mammary gland [24]. Further analysis of milk FA composition showed that the proportion of SFA, especially SMCFA (C6:0, C8:0, C10:0 and C12:0), was significantly increased with the addition of inulin, but the ratio of C18:0 and C22:0 were decreased. The increase in SMCFA might due to the increase in the concentration of acetate and butyrate in the rumen, which are the substrates for de novo synthesis of milk FA by mammary epithelial cells [25], while about half of the $\mathrm{C} 16: 0$ and other long-chain FA in milk fat are directly absorbed from blood lipids [25]. On the other hand, the results of metabolomics in our study revealed that the supplementation of inulin significantly downregulated the lipid metabolism in dairy cows. The increase in the ratio of SFA leaded to a decrease in the ratio of UFA [25]. The decrease in C18:1 trans-9 and C18:2 cis6 might be related to the hydrogenation of rumen microorganisms, which could reduce the toxicity of UFA [26]. In addition, studies reported that milk fat percentage was negatively correlated with trans-C18:1 in milk [27]. Trans-FAs (TFAs) could restrain the expression of milk fat synthase gene, such as acetylcoenzyme A carboxylase and FA synthase [27].

In accordance with the improvement effect of inulin on intestinal flora in humans and monogastric animals, in the current study, inulin also showed a positive effect on ruminal microbiome through increasing the relative abundance and diversities of rumen microflora. The possible reasons may due to the increase of feed intake and the optimized rumen microbiota structure induced by inulin $[14,28]$. In the current study, the supplementation of inulin significantly increased the relative abundance of Bacteroides, which could regulate nutrient absorption and metabolism of exogenous substances, such as polysaccharides [29]. In addition, inulin intake mainly increased the relative abundance of several probiotics and SCFA-producing bacteria in the rumen. Muribaculaceae (also called S24-7) belonged to Bacteroides has been found to act as a probiotic in multiple studies, or related with the innate immune system [30]. For example, homeostatic IgA responses could target Muribaculaceae resided in small intestine [30]. In addition, the relative abundance of the Muribaculaceae was improved with the increase of dietary fiber levels [31]. Besides, Muribaculaceae has the function of degrading complex carbohydrates 
and is positively correlated with the concentration of intestinal acetate, propionate and butyrate [31]. Another interesting bacteria with significant change in abundance induced by inulin addition is Butyrivibrio. Butyrivibrio was the main butyrate-producing bacteria, followed by LA [32]. As a similar shortchain fatty acid producer, the main products of Prevotellaceae_NK3B31_group fermented FOS are acetate and a small amount of isobutyrate, isovalerate and LA [31]. Furthermore, Acetitomaculum was another well-known acetate and LA producing bacteria [33]. The increase of these bacteria abundance in rumen was in accordance with the elevated SCFA ratio in blood, which might further promote milk fat synthesis [34]. On the other hand, the increase of dietary fiber level might promote the growth of a series of cellulolytic bacteria in the gastrointestinal tract. Eubacterium_hallii_group belonged to the Lacetospirillum family could also degrade fructose and cellobiose, which contributed to the formation of propionate and LA in rumen [35]. In addition, Eubacterium_hallii_group could convert glycerol to 3-

hydroxypropionaldehyde, and formed a multi-compound system called reuterin in aqueous solution, which has antibacterial properties [35]. Both Treponema and Saccharofermentans could degrade cellulose and hemicellulose to generate large amounts of propionate $[36,37]$. Therefore, the significant increase of the above bacteria may be the main reason of the increase of propionate concentration in rumen and milk lactose rate.

On the contrary, the significantly decreased ruminal microflora induced by inulin were mainly several conditional pathogens, high-abundant bacteria after high-fat feeding and microorganisms involved in FA hydrogenation. Escherichia-Shigella, Anaerobiospirillum and Clostridia have been reported to cause gastrointestinal infection or inflammation $[38,39,40]$. Similarly, as a conditioned pathogens, Erysipelotrichaceae that resided in the mouth and intestine of animals could result in endogenous infections, and significantly increased in the intestinal tract of colon cancer patients. ${ }^{41}$ The current study showed that the above bacteria were positively correlated with milk SCC and negatively correlated with milk fat, protein and lactose, which implicated the adverse effects of them on milk compositions. Moreover, Erysipelotrichaceae, Clostridia, Syntrophococcus and RF39 have been reported to increase significantly when feeding a high-fat diet. Studies have shown that the abundance of Erysipelotrichaceae in the intestines of rats with long-term high-fat or Western diets was 2.5 times higher than that of the control group [41]. Martinez et al. proved that there was a positive correlation between the level of Erysipelotrichaceae and host cholesterol metabolites [42]. The increase in the abundance of Clostridia in the intestines of rats with a high-fat diet might be related to low fiber content [40]. Additionally, the study on growth requirements of Syntrophococcus in rumen fluid showed that TG and phospholipids could promote the proliferation of Syntrophococcus [43]. RF39 belongs to the Tenericutes phylum, while a highfat diet could increase the abundance of Tenericutes, which made it easier for the host to absorb lipids [44]. On the other hand, Selenomonas and Bacteroidales_BS11_gut_group were reported to be involved in the hydrogenation of $\mathrm{C} 18: 3 \mathrm{n} 3$ and $\mathrm{C} 18: 2 \mathrm{n} 6 \mathrm{c}$, respectively. The probable reasons were that they provided energy substance, ATP, for the hydrogenation of these two FAs $[45,46]$.

In summary, after supplementation of inulin in dietary, the decrease of rumen $\mathrm{pH}$ and the increase of VFAs concentration might be attributed to the proliferation of SCFAs-producing bacteria. On the other 
hand, the acidic environment in rumen effectively inhibited the proliferation of pathogens, which might be another reason for the decrease in milk SCC. Besides, more microorganisms increase the utilization of protein in the rumen and further increase the milk protein percentage, which might also explain the negative correlation between the dominant bacteria in the inulin group and $\mathrm{MUN}$ and $\mathrm{NH}_{3}-\mathrm{N}$.

The result of metabolomics further revealed that supplementation of inulin significantly affected the amino acid and lipid metabolism in dairy cows. In the current study, the decrease in the level of LysoPC(18:1(9Z)), LysoPC(16:0) and LysoPC(18:2(9Z,12Z)) in the rumen of cows fed inulin downregulated the glycerophospholipid and choline metabolism pathways. Lysophosphatidylcholine (LysoPC) is derived from the hydrolysis of lecithin or the oxidation of very low density lipoprotein (VLDL). It was reported that inulin could reduce the number of VLDL particles in plasma and inhibit the expression of enzymes involved in lipid synthesis, such as acetyl-CoA carboxylase, fatty acid synthase and malic enzyme in the liver, which could reduce the de novo synthesis of FAs and the levels of TG and cholesterol in serum, [47] which was consist with our results. Another possible reason that the decrease in LysoPCs was that inulin could promote the proliferation of SCFA-producing bacteria and reduce the $\mathrm{pH}$ of the rumen, which led to the reduction of solubility of bile acids and increase in the excretion of bile acids and steroids in feces [48]. This might explain the negative correlation between LysoPC (18:1 (9Z)), LysoPC (16:0), LysoPC (18:2 (9Z, 12Z)), and Muribaculaceae, Prevotellaceae_NK3B31_group and other SCFAproducing bacteria. However, in our study, the decline of the level of LysoPCs were not showed a significant negative correlation with milk fat. The possible reason was that the LysoPCs was not a key factor affecting milk fat synthesis. On the other hand, LysoPCs have been reported to have proinflammatory activity, which was related to cytosolic phospholipase A2 mediating phosphatidylcholine (PCs) to produce arachidonic acid [49]. This might be the reason that the positive correlation between LysoPC(18:1(9Z)), LysoPC(16:0), LysoPC(18:2(9Z,12Z)) and milk SCC as well as several pathogens abundance, including Escherichia-Shigella and Anaerobiospirillu, etc., observed in this study. Other downregulated lipids included Phenylmethylglycidic ester and 8-Methylnonenoate.

Phenylmethylglycidic ester was a sweet, fatty, and floral compound [50]. However, its metabolic pathway was needed to be further explored. 8-methyl-nonenoic acid was a long-chain fatty acid derivative from leucine/valine pathway, and also a component of fatty acyl groups [51]. In the current study, the decrease of 8-methyl-nonenoic acid level was consistent with the decrease in the proportion of long-chain fatty acids in milk. Therefore, the declined levels the above ruminal metabolites might imply the potential of inulin to downregulate the lipid metabolism in dairy cows.

$\mathrm{N}$-Acetylcadaverine is the acetylated form of the polyamine cadaverine, which is formed by the decarboxylation of lysine [52]. In addition, N-Acetylcadaverine was mainly a metabolite of Escherichia coli. Also, it has been found that there were variants of the N-Acetylcadaverine in Bacillus species [52]. The accumulation of a large amount of N-Acetylcadaverine in the body was related to the production of bacteriocins and toxin activity, which also had a specific induction effect on inflammatory factors [52]. This might illustrate the positive correlation between N-Acetylcadaverine and milk SCC, EscherichiaShigella, Erysipelotrichaceae (Bacillus class). 
The AAs in the rumen were mainly derived from the degradation of feed proteins by rumen microorganisms. In the present study, the significant increase in the levels of L-Lysine, L-Proline, LPhenylalanine and L-Tyrosine in cows fed inulin might be attributed to the proliferation of the beneficial symbiotic bacteria in rumen, which accelerated the decomposition of dietary protein and synthesis of MCP, and further promoted the synthesis of milk protein [17]. Consistent with Lykos et al., the increasing of nonstructural carbohydrates in the rumen could increase the level of non-essential AAs in the blood [53]. On the other hand, inulin regarded as a prebiotics could provide substrates for intestinal flora to generate SCFAs [54]. Davila et al. proposed that AAs could synthesize SCFAs by the prebiotic-activated intestinal flora [54]. Lysine, phenylalanine and tyrosine could be used as precursors of acetate and butyrate. Butyrate was derived from glutamic acid and lysine, and propionate was synthesized from alanine and threonine [54]. Meanwhile, isoacids, including isobutyrate, isovalerate and valerate, were generated by degradation of proline [55]. This might explain the positive correlation between L-Lysine, LProline, L-Phenylalanine and L-Tyrosine and SCFA-producing bacteria. Moreover, the positive correlation between L-Lysine, L-Proline, L-Phenylalanine and L-Tyrosine, and milk fat might be due to the fact that AAs could enhance the uptake efficiency of milk fat precursors by mammary gland and thus regulate the content of VFAs in the udder, which promoted the de novo synthesis of FAs in the mammary gland [56]. Consistent with Maxin et al. (2011), the increase of AAs content in milk could promote the milk fat production. Among them, the content of short- and medium-chain FAs (C6:0-C14:0) tended to increase, while the C16:0, C18:0, C18:2 and other long-chain FAs were decreased [57].

In the present study, the increase of uracil upregulated the nucleic acid metabolism, which might indicate that supplementation of inulin might promote the synthesis of rumen MCP [17]. Additionally, the decomposed product of uracil was $\beta$-alanine, which could further synthesize propionate [58]. This might explain the positive correlation between uracil and lactose. Deltonin and daidzein were reported to have anti-tumor activity and inhibit inflammatory factors in serum $[59,60]$. The negative correlation between deltonin, daidzein and milk SCC might reveal the enhancement of immune and disease resistance in dairy cows. However, the effect of inulin on the secondary metabolites of plants in the rumen still needs to be further explored.

Throughout the study, the effects of supplementation of inulin in dietary on lactation performance and rumen fermentation characteristics in dairy cows might be mainly through the influence on the ruminal bacteria profile, which further affected the kinds and concentrations of rumen metabolites. The correlation among them indicated that the shifts in composition and abundance of ruminal microbiome as well as the concentration and activity of rumen metabolites caused by the increase of dietary fiber intake might be a key factor affecting performance and physiological indicators in dairy cows.

\section{Conclusion}

The supplementation of inulin in diets of dairy cows could increase the abundance of beneficial symbiotic bacteria and SCFAs-producing bacteria in the rumen, meanwhile, it promoted AAs synthesis and metabolism, and inhibited lipid metabolism. The shift of rumen microorganisms and metabolites 
further resulted in the increase in concentration of VFAs in the rumen, while decline the level of TC and TG in serum. The change of these precursor of milk composition might the reason that the increase in milk yield, protein, lactose rate and SFAs proportion in milk, but decrease in the percentage of PUFAs in milk. However, the metabolic processes and mechanism of the action of inulin in the rumen of dairy cows merit further investigation.

\section{Abbreviations}

FOS, fructo-oligosaccharide; SCFAs, short-chain fatty acids; LA, lactic acid; TG, triglycerides; $\mathrm{NH}_{3}-\mathrm{N}$, ammoniacal nitrogen; $A / P$, the ratio of acetate to propionate; BW, body weight; DIM, days in milk; SCC, somatic cell counts; TMR, total mixed rations; DMI, dry matter intake; MUN, urea nitrogen; FAs, fatty acids; SMCFA, short and medium-chain fatty acids; LCFA, long-chain fatty acids; TFAs, trans-fatty acids; VFAs, volatile fatty acids; RUN, rumen urea nitrogen; QC, quality control; OTU, operational taxonomic units; PCoA, principal co-ordinates analysisand; NMDS, non-metric multidimensional scaling; LEfSe, linear discriminant analysis effect size; KW, Kruskal-Wallis; FDR, false discovery rate; LDA, linear discriminant analysis; RDP, ribosomal database project; $\mathrm{RT}$, retention time; $\mathrm{M} / \mathrm{Z}$, mass-to-charge ratio; RSD, relative standard deviation; HMDB, human metabolome database; PCA, principal component analysis; OPLS-DA, orthogonal least partial squares discriminant analysis; FC, fold change; VIP, variable important in projection; $\mathrm{HCA}$, hierarchical clustering analysis; $\mathrm{ROC}$, receiver operator characteristic; $\mathrm{R}$, correlation coefficient; ECM, energy corrected milk; FCM, fat corrected milk; F/P, fat to protein ratio; SFA, saturated fatty acid; UFA, unsaturated fatty acid; PUFA, polyunsaturated fatty acids; TIC, total ion chromatograms; RPT, response permutation testing; ROC, receiver operator characteristic curve; AUC, area under curve; MCP, microbial protein; AAs, amino acids; LysoPC, lysophosphatidylcholine; VLDL, very low-density lipoprotein; PCs, phosphatidylcholine; TC, total cholesterol; TG, triglyceride; SEM, standard error of mean.

\section{Declarations}

\section{Acknowledgments}

We thank the Beijing Key Laboratory for Dairy Cow Nutrition, Beijing University of Agriculture, Beijing, China for providing the experimental equipment.

\section{Authors' contributions}

The experimental scheme was designed by YW. The inulin used in this study was provide by JL. DH and WY participated in the experiment process and assisted in sampling. The analysis of experimental data and the making of charts were completed by $\mathrm{YW}$. YW completed the initial draft, $\mathrm{XN}$ and $\mathrm{YZ}$ completed the overall modification of the manuscript. $Y Z$ and $\mathrm{HW}$ improved and polished the language of the article. $\mathrm{LJ}$ and JY provided the necessary experimental equipment and key guidance during the experiment process, while BX was responsible for the overall content and framework of the paper.

\section{Funding}


This study was funded by the National Key R\&D Program of China (Grant No. 2016YFD0700205区 2018YFD0500703, 2017YFD0701604) and Beijing Dairy Industry Innovation Team (bjcystx-ny-1).

\section{Availability of data and materials}

All data generated or analyzed during this study are included in this published article (and supplementary information files).

\section{Ethics approval}

All experimental designs and protocols were approved by the Animal Ethics Committee of the Chinese Academy of Agricultural Sciences (Beijing, China) (approval number: IAS-2019-9) and were in accordance with the recommendations of the academy's guidelines for animal research.

\section{Consent for publication}

Not applicable.

\section{Competing interests}

The authors declare that they have no competing interests.

\section{Author details}

${ }^{1}$ State Key Laboratory of Animal Nutrition, Institute of Animal Science, Chinese Academy of Agricultural Sciences, Beijing, 100193, China. ${ }^{2}$ Beijing Key Laboratory for Dairy Cow Nutrition, Beijing University of Agriculture, Beijing, 102206, China. ${ }^{3}$ College of Animal Science and Technology, Northwest A\&F University, Yangling 712100, P. R. China. ${ }^{4}$ Langfang Academy of Agriculture and Forestry, Langfang, 065000, China

\section{References}

1. Saengthongpinit W, Sajjaanantakul T. Influence of harvest time and storage temperature on characteristics of inulin from Jerusalem artichoke (Helianthus tuberosus L.) tubers. Postharvest Biol Tec. 2005; 37 (1): 93-100. https://doi.org/ 10.1016/j.postharvbio.2005.03.004.

2. Roberfroid MB. Inulin-type fructans: functional food ingredients. J Nutr. 2007; 137 (11): 2493S2502S. https://doi.org/10.1201/9780203504932.

3. Niness KR. Inulin and oligofructose: what are they? J Nutr 1999; 129 (7): 1402S-06S. https://doi.org/ 10.1038/sj.ijo.0800961.

4. Ronkart S, Blecker C, Fougnies C, Herck JCV, Wouters J, Paquot M. Determination of physical changes of inulin related to sorption isotherms: an x-ray diffraction, modulated differential scanning calorimetry and environmental scanning electron microscopy study. Carbohyd Polym. 2006; 63 (2): 210-7. https://doi.org/10.1016/j.carbpol.2005.08.030. 
5. Schley PD, Field CJ. The immune-enhancing effects of dietary fibres and prebiotics. Brit J Nutr. 2002; 87 (2): 221-30. https://doi.org/10.1079/BJNBJN/2002541.

6. Chen K, Chen H, Faas MM, De Haan BJ, Li J, Xiao P, et al. Specific inulin-type fructan fibers protect against autoimmune diabetes by modulating gut immunity, barrier function, and microbiota homeostasis. Mol Nutr Food Res. 2017; 61 (8): 1601006. https://doi.org/10.1002/mnfr.201601006.

7. Lopez HW, Coudray C, Levrat-Verny MA, Feillet-Coudray C, Christian D, Christian R. Fructooligosaccharides enhance mineral apparent absorption and counteract the deleterious effects of phytic acid on mineral homeostasis in rats. J Nutr Biochem. 2000; 11(10): 500-08. https://doi.org/ 10.1016/S0955-2863(00)00109-1.

8. Williams CM. Effects of inulin on lipid parameters in humans. J Nutr. 1999; 129 (7): 1471-73. https://doi.org/10.1038/sj.ijo.0800961.

9. Causey JL, Feirtag JM, Gallaher DD, Tungland BC, Slavin JL. Effects of dietary inulin on serum lipids, blood glucose and the gastrointestinal environment in hypercholesterolemic men. Nutr Res. 2000; 20 (2): 191-201. https://doi.org/10.1016/S0271-5317(99)00152-9.

10. Biggs DR, Hancock KR. In vitro digestion of bacterial and plant fructans and effects on ammonia accumulation in cow and sheep rumen fluids. J Gen Appl Microbiol. 1998; 44(2): 167-71. https://doi.org/10.2323/jgam.44.167.

11. Zhao XH, Gong JM, Zhou S, Liu CJ, Qu MR. The effect of starch, inulin, and degradable protein on ruminal fermentation and microbial growth in rumen simulation technique. Ital J Anim Sci. 2016; 13 (1): 189-95. https://doi.org/10.4081/ijas.2014.3121.

12. Gndz, N. Effects of chicory inulin on ruminal fermentation in vitro. Ankara Üniv Vet Fak Derg. 2009; 56(3): 171-75.

13. Umucalilar HD, Glen N, Hayirli A, Alata MS. Potential role of inulin in rumen fermentation. Rev Med Vet. 2010; 161(1): 3-9. https://doi.org/10.1590/S1516-35982010000100030.

14. Tian K, Liu J, Sun Y, Wu Y, Chen J, Zhang R, et al. Effects of dietary supplementation of inulin on rumen fermentation and bacterial microbiota, inflammatory response and growth performance in finishing beef steers fed high or low-concentrate diet. Anim Feed Sci Tech 2019; 258: 114229. https://doi.org/10.1016/j.anifeedsci.2019.114299.

15. Wang B, Tu Y, Zhao SP, Hao YH, Liu JX, Liu FH, et al. Effect of tea saponins on milk performance, milk fatty acids, and immune function in dairy cow. J Dairy Sci. 2017; 100 (10): 8043-52. https://doi.org/10.3168/jds.2016-12425.

16. González-Córdova AF, Vallejo-Cordoba B. Quantitative determination of short-chain free fatty acids in milk using solid-phase microextraction and gas chromatography. J Agr Food Chem. 2001; 49 (10): 4603-08. https://doi.org/10.1021/jf010108d.

17. Liu C, Wu H, Liu S, Chai S, Meng Q, Zhou Z. Dynamic alterations in yak rumen bacteria community and metabolome characteristics in response to feed type. Front Microbiol. 2019; 10, 1-19. https://doi.org/10.3389/fmicb.2019.01116. 
18. Hue WL, Liu JX, Ye JA. Effects of tea saponin on rumen fermentation in vitro. Anim Feed Sci Tech. 2005; 120 (4): 333-39. https://doi.org/10.1016/j.anifeedsci.2005.02.029.

19. Han X, Yang Y, Yan H, Wang X, Qu L, Chen Y. Rumen bacterial diversity of 80 to 110-day-old goats using 16s rRNA sequencing. Plos One. 2015; 10 (2): e0117811. https://doi.org/10.1371/journal.pone.0117811.

20. Ogunade I, Schweickart H, Mccoun M, Cannon K, Mcmanus C. Integrating 16s rRNA sequencing and LC-MS-based metabolomics to evaluate the effects of live yeast on rumen function in beef cattle. Animals. 2019; 9 (1): 1-14. https://doi.org/10.3390/ani9010028.

21. Artegoitia VM, Foote AP, Lewis RM, Freetly HC. Rumen fluid metabolomics analysis associated with feed efficiency on crossbred steers. Sci Rep. 2017; 7(1): 2864-78. https://doi.org/10.1038/s41598017-02856-0

22. Samanta AK, Natasha J, Senani S, Kolte AP, Manpal S. Prebiotic inulin: useful dietary adjuncts to manipulate the livestock gut microflora. Braz J Microbiol. 2013; 44 (1): 1-14. https://doi.org/10.1590/S1517-83822013005000023.

23. Emery RS. Feeding for increased milk protein1. J Dairy Sci. 1978; 61(6): 825-28. https://doi.org/ 10.3168/jds.S0022-0302(78)83656-X.

24. Sunehag A, Tigas S, Haymond MW. Contribution of plasma galactose and glucose to milk lactose synthesis during galactose ingestion. J Clin Endocr Metab. 2003; 88 (1): 225-29. https://doi.org/10.1210/jc.2002-020768

25. Cozma A, Miere D, Filip L, Andrei S, Banc R, Loghin F. A review of the metabolic origins of milk fatty acids. Not Sci Biol. 2013; 5(3): 270-74. https://doi.org/10.15835/nsb.5.3.9120.

26. Lock AL, Bauman DE. Modifying milk fat composition of dairy cows to enhance fatty acids beneficial to human health. Lipids. 2004; 39 (12): 1197-1206. https:// doi.org/10.1007/s11745-004-1348-6.

27. Matitashvili EA, Bauman DE. Effeet of different isomers of C18:1 and C18:2 fatty acids on lipogenesis in bovine mammary eipthelial cells. J Dairy Sci. 2000; 83 (suppl.1): 165.

28. Chambers ES, Byrne CS, Morrison DJ, Murphy KG, Preston T, Tedford C, et al. Dietary supplementation with inulin-propionate ester or inulin improves insulin sensitivity in adults with overweight and obesity with distinct effects on the gut microbiota, plasma metabolome and systemic inflammatory responses: a randomised cross-over trial. Gut. 2019; 1-9. https://doi.org/10.1136/gutjnl-2019-318424.

29. Ivarsson E, Roos S, Liu HY, Lindberg JE. Fermentable non-starch polysaccharides increases the abundance of bacteroides-prevotella-porphyromonas in ileal microbial community of growing pigs. Animal. 2014; 8 (11): 1777-87. https://doi.org/10.1017/S1751731114001827.

30. Bunker JJ, Flynn TM, Koval JC, Shaw DG, Meisel M, McDonald BD, et al. Innate and Adaptive Humoral Responses Coat Distinct Commensal Bacteria with Immunoglobulin A. Immunity. $2015 ; 43$ (3): 541-53. https://doi.org/10.1016/j.immuni.2015.08.007.

31. Zhao L, Zhang F, Ding X, Wu G, Lam YY, Wang X, et al. Gut bacteria selectively promoted by dietary fibers alleviate type 2 diabetes. Science. 2018; 359 (6380): 1151-56. https://doi.org/10.1126/ 
science.aao5774.

32. Kopecny J. Butyrivibrio hungatei sp. nov. and Pseudobutyrivibrio xylanivorans sp. nov. butyrateproducing bacteria from the rumen. Int J Syst Evol Micr. 2003; 53 (1): 201-09. https://doi.org/10.1099 /ijs.0.02345-0.

33. Rees EMR, David L, Williams AG. The effects of co-cultivation with the acetogen Acetitomaculum ruminis on the fermentative metabolism of the rumen fungi neocallimastix patriciarum and neocallimastix sp. strain I2. Fems Microbiology Letters. 1995; (1-2): 175-180. https://doi.org/10.1016 /0378-1097(95)00367-E.

34. Lofgren PA, Warner RG. Influence of various fiber sources and fractions on milk fat percentage. J Dairy Sci. 1970; 53 (3): 296-304. https://doi.org/10.3168/jds.S0022-0302(70)86200-2.

35. Engels C, Ruscheweyh HJ, Beerenwinkel N, Lacroix C, Schwab C. The common gut microbe Eubacterium hallii also contributes to intestinal propionate formation. Front Microbiol. $2016 ; 7$ (13): 1-12. https://doi.org/10.3389/fmicb.2016.00713.

36. Binek M, Szynkiewicz ZM. Physiological properties and classification of strains of Treponema sp. isolated from pigs in poland. Comp Immunol Microb. 1984; 7(3-4): 141-48. https://doi.org/10.1016/ 0147-9571(84)90019-5.

37. Perea K, Perz K, Olivo SK, Williams A, Yeoman CJ. Feed efficiency phenotypes in lambs involve changes in ruminal, colonic, and small-intestine-located microbiota. J Anim Sci. 2017; 95(6): 258592. https://doi.org/10.2527/jas2016.

38. Chen L, Wang W, Zhou R, Ng SC, Li J, Huang M, et al. Characteristics of fecal and mucosa-associated microbiota in chinese patients with inflammatory bowel disease. Medicine. 2014; 93(8): e51-60. https://doi.org/10.1097/MD.0000000000000051.

39. Misawa N, Kawashima K, Kondo F, Kushima E, Vandamme P. Isolation and characterization of Campylobacter, Helicobacter, and Anaerobiospirillum strains from a puppy with bloody diarrhea. Vet Microbiol. 2002; 87(4): 353-64. https://doi.org/10.1016/S0378-1135(02)00086-X

40. De La Serre CB, Ellis CL, Lee J, Hartman AL, Rutledge JC, Raybould HE. Propensity to high-fat dietinduced obesity in rats is associated with changes in the gut microbiota and gut inflammation. AM $J$ Physiol-Gastr L. 2010; 299 (2): G440-G48. https://doi.org/10.1152/ajpgi.00098.2010.

41. Kaakoush NO. Insights into the role of Erysipelotrichaceae in the human host. Front Cell Infect Microbiol. 2015; 5 (84):1-4. https://doi.org/10.3389/fcimb.2015.00084.

42. Martínez I, Wallace G, Zhang C, Legge R, Benson AK, Carr TP, et al. Diet-induced metabolic improvements in a hamster model of hypercholesterolemia are strongly linked to alterations of the gut microbiota. Appl Environ Microbiol. 2009; 75: 4175-84. https://doi.org/10.1128/AEM.00380-09.

43. Doré J, Bryant MP. Lipid growth requirement and influence of lipid supplement on fatty acid and aldehyde composition of Syntrophococcus sucromutans. Appl Environ Microbiol. 1989; 55(4): 92733. https://doi.org/10.1002/bit.260330921.

44. Niu Q Li P, Hao S, Hang Y, Kim SW, Li H, et al. Dynamic distribution of the gut microbiota and the relationship with apparent crude fiber digestibility and growth stages in pigs. Sci Rep. 2015; 5 (9938): 
9938. https://doi.org/10.1038/srep09938.

45. Benz J, Wolf C, Rüdiger Wolfhart. Chlorophyll biosynthesis: hydrogenation of geranylgeraniol. Plant Sci Lett. 1980; 19 (3): 225-30. https://doi.org/10.1016/0304-4211 (80)90076-0.

46. Solden L, Hoyt DW, Collins W, Plank J, Daly R, Erik CH, et al. New roles in hemicellulosic sugar fermentation for the uncultivated Bacteroidetes family BS11. ISME. J. 2016; 11 (3): 691-703. https://doi.org/10.1038/ismej.2016.150.

47. Beylot, Michel. Effects of inulin-type fructans on lipid metabolism in man and in animal models. Brit. J. Nutr. 2005; 93(S1): S163-S68. https://doi.org/10.1079/BJN20041339.

48. Roberfroid MB, Delzenne NM. Dietary fructans. Annu. Rev. Nutr. 1998; 18 (1): 117-43. https:// doi.org/10.1146/annurev.nutr.18.1.117.

49. You JC, Yang J, Fang RP, Hu N, Ye LH. Analysis of phosphatidylcholines (PCs) and lysophosphatidylcholines (LysoPCS) in metastasis of breast cancer cells. Prog Biochem Biophys. 2015; 42 (6): 563-73. https://doi.org/10.16476/j.pibb.2015.0031.

50. Allen CFH, Vanallan J. Phenylmethylglycidic Ester. Org Syn Coll. American Cancer Society. 2003; 3 (733): 1944-55.

51. Markai S, Marchand PA, Mabon F, Baguet E, Billault I, Robins RJ. Natural deuterium distribution in branched-chain medium-length fatty acids is nonstatistical: a site-specific study by quantitative ${ }^{2} \mathrm{H}$ NMR spectroscopy of the fatty acids of capsaicinoids. Chembiochem. 2010; 3(2-3): 212-18. https://doi.org/10.1002/1439-7633 (20020301)3:2/3<212::AID-CBIC212>3.0.C0;2-R.

52. Ma WC, Chen KQ, Li Y, Wang X, Ouyang PK. Advances in cadaverine bacterial production and its applications. Engineering. 2017; 3: 308-17. https://doi.org/10.1016/J.ENG.2017.03.012.

53. Lykos T, Varga GA. Varying degradation rates of total nonstructural carbohydrates: effects on nutrient uptake and utilization by the mammary gland in high producing Holstein cows. J Dairy Sci. 1997; 80 (12): 3356-67. https://doi.org/10.3168/jds.S0022-0302(97)76311-2.

54. Davila AM, Blachier F, Gotteland M, Andriamihaja M, Benetti PH, Sanz Y, et al. Intestinal luminal nitrogen metabolism: role of the gut microbiota and consequences for the host. Pharmacol Res. 2013; 68(1): 95-107. https://doi.org/10.1016/j.phrs.2012.11.005.

55. Andries JI, Buysse FX, Brabander DLDe, Cottyn BG. Isoacids in ruminant nutrition: Their role in ruminal and intermediary metabolism and possible influences on performances-A review. Anim Feed Sci Tech. 1987; 18 (3): 169-80. https://doi.org/10.1016/0377-8401 (87)90069-1.

56. Cant JP, Trout DR, Qiao F, Purdie NG. Milk synthetic response of the bovine mammary gland to an increase in the local concentration of arterial glucose. J Dairy Sci. 2002; 85(3): 494-503. https://doi. org/10.3168/jds.S0022-0302 (02)74100-3.

57. Maxin G, Rulquin H, Glasser F. Response of milk fat concentration and yield to nutrient supply in dairy cows. Animal. 2011; 5(8):1299-1310. https://doi.org/10.1017/S1751731111000206.

58. West TP, Traut TW, Shanley MS, O'Donovan GA. A salmonella typhimurium strain defective in uracil catabolism and $\beta$-alanine synthesis. Microbiology. 1985; 131(5): 1083-90. 
59. Tong QY, Qing Y, Shu D, He Y, Zhao YL, Li Y, et al. Deltonin, a steroidal saponin, inhibits colon cancer cell growth in vitro and tumor growth in vivo via induction of apoptosis and antiangiogenesis. Cell Physiol Biochem. 2011; 27(3-4): 233-42. https://doi.org/10.1159/000327949.

60. Shen JL, Li N, Zhang X. Daidzein ameliorates dextran sulfate sodium-induced experimental colitis in mice by regulating NF-KB signaling. J Environ Pathol Toxicol Oncol. 2019; 38 (1): 29-39. https://doi. org/10.1615/JEnvironPatholToxicolOncol.2018027531.

61. National Research Council (NRC). Nutrient Requirements of Dairy Cattle: Seventh Revised Edition. The National Academies Press, Washington, DC. 2001; 4: 27-28.

62. Sjaunja LO, Baevre L, Junkkarinen L, Pedersen J, Setälä J. A Nordic proposal for an energy corrected milk (ECM) formula. Paper presented at Proceedings of the 27th Biennial Session of the International Committee for Animal Recording (ICAR). 1990; 2-6.

63. Gaines WL. The energy basis of measuring milk yield in dairy cows. Illinois agricultural experiment station bulletin 308. Urbana: University of Illinois. 1928, 436-38.

\section{Tables}

Table 1. Effects of inulin supplementation in dietary on milk yield and compositions

\begin{tabular}{|lllll|}
\hline Items & $\mathbf{C}(\mathbf{n}=\mathbf{8})$ & $\mathbf{I}(\mathbf{n}=\mathbf{8})$ & SEM & $P$-value \\
\hline DMI & $23.9^{\mathrm{b}}$ & $25.1^{\mathrm{a}}$ & 0.11 & 0.003 \\
\hline BW & 551 & 554 & 1.5 & 0.079 \\
\hline Milk yield $(\mathrm{kg} / \mathrm{d})$ & $34.4^{\mathrm{b}}$ & $37.2^{\mathrm{a}}$ & 0.55 & 0.001 \\
\hline ECM $(\mathrm{kg} / \mathrm{d})$ & $34.0^{\mathrm{b}}$ & $37.8^{\mathrm{a}}$ & 0.79 & $<0.001$ \\
\hline FCM $(\mathrm{kg} / \mathrm{d})$ & $34.8^{\mathrm{b}}$ & $38.0^{\mathrm{a}}$ & 0.70 & $<0.001$ \\
\hline Milk fat $(\%)$ & 4.08 & 4.14 & 0.034 & 0.075 \\
\hline Milk protein $(\%)$ & $3.12^{\mathrm{b}}$ & $3.39^{\mathrm{a}}$ & 0.068 & 0.032 \\
\hline F/P & $1.31^{\mathrm{a}}$ & $1.22^{\mathrm{b}}$ & 0.033 & 0.027 \\
\hline Milk lactose $(\%)$ & $4.85^{\mathrm{b}}$ & $5.19^{\mathrm{a}}$ & 0.066 & 0.004 \\
\hline MUN (mmol/L) & $5.99^{\mathrm{a}}$ & $5.18^{\mathrm{b}}$ & 0.186 & 0.023 \\
\hline SCC $\left(\times 10^{3} \mathrm{cells} / \mathrm{mL}\right)$ & $340^{\mathrm{a}}$ & $302^{\mathrm{b}}$ & 14.1 & 0.036 \\
\hline
\end{tabular}

$\mathrm{C}=$ control group; $\mathrm{I}$ = inulin group; $\mathrm{DMI}=$ dry matter intake; $\mathrm{BW}=$ body weight; $\mathrm{ECM}$ = energy corrected milk; $F C M=$ fat corrected milk; $F / P=$ fat to protein ratio; $M U N=$ milk urea nitrogen; $S C C=$ somatic cell 
counts; SEM = standard error of mean; ${ }^{a}$ b within a row, different letters means differed significantly $(P<$ 0.05).

$\operatorname{DMI}(\mathrm{kg} / \mathrm{d})=\left(0.372 \times\right.$ fat corrected milk $+0.0968 \times$ live weight $\left.{ }^{0.75}\right) \times\left(1-\mathrm{e}^{(-0.192 \times(\text { week of lactation }+3.67))}\right)$ [61];

$\operatorname{ECM}(\mathrm{kg} / \mathrm{d})=$ milk yield $(\mathrm{kg} / \mathrm{d}) \times(383 \times f a t \%+242 \times$ protein $\%+783.2) / 3140$ [62];

FCM $(\mathrm{kg} / \mathrm{d})=0.4 \times$ milk yield $(\mathrm{kg} / \mathrm{d})+15 \times$ milk yield $(\mathrm{kg} / \mathrm{d}) \times \%$ fat [63]

Table 2. Effect of inulin supplementation in dietary on milk fatty acids compositions 


\begin{tabular}{|c|c|c|c|c|c|}
\hline Items (\%) & Name & $\begin{array}{l}C \\
8)\end{array}$ & $\begin{array}{l}I(n= \\
8)\end{array}$ & SEM & $\begin{array}{l}P \\
\text { value }\end{array}$ \\
\hline SFA & & $65.9^{\mathrm{b}}$ & $70.1^{\mathrm{a}}$ & 1.37 & $<0.001$ \\
\hline C4:0 & Butyric acid & 3.11 & 3.38 & 0.064 & 0.99 \\
\hline C6:0 & Methyl hexanoate & $2.08^{\mathrm{b}}$ & $2.72^{\mathrm{a}}$ & 0.060 & 0.034 \\
\hline C8:0 & Methyl octanoate & $1.28^{b}$ & $1.37^{\mathrm{a}}$ & 0.042 & 0.006 \\
\hline C10:0 & Capric acid & $2.81^{b}$ & $3.02^{\mathrm{a}}$ & 0.088 & 0.038 \\
\hline C11:0 & Methyl undecanoate & 0.040 & 0.069 & 0.0103 & 0.175 \\
\hline C12:0 & Dodecane lauric acid & $2.58^{\mathrm{b}}$ & $3.64^{\mathrm{a}}$ & 0.179 & 0.001 \\
\hline C13:0 & Methyl tridecanote & 0.10 & 0.11 & 0.006 & 0.295 \\
\hline C14:0 & Myristoic acid & 9.65 & 9.97 & 0.368 & 0.546 \\
\hline C15:0 & Methyl pentadecanoate & 0.090 & 0.090 & 0.0305 & 0.427 \\
\hline C16:0 & Palmitic acid & $33.2^{\mathrm{b}}$ & $37.3^{a}$ & 0.65 & 0.001 \\
\hline C17:0 & Methyl heptadecanoate & 0.47 & 0.43 & 0.007 & 0.54 \\
\hline C18:0 & Octadecanoic acid & $9.77^{\mathrm{a}}$ & $7.45^{\mathrm{b}}$ & 0.421 & 0.002 \\
\hline C2O:0 & Eicosanoic acid & 0.095 & 0.068 & 0.0059 & 0.273 \\
\hline $\mathrm{C} 21: 0$ & Methyl heneicosanoate & 0.42 & 0.33 & 0.015 & 0.101 \\
\hline $\mathrm{C} 22: 0$ & Behenic acid & $0.17^{a}$ & $0.13^{b}$ & 0.013 & 0.031 \\
\hline UFA & & $27.8^{a}$ & $26.4^{\mathrm{b}}$ & 0.65 & 0.041 \\
\hline MUFA & & 24.1 & 23.3 & 0.64 & 0.066 \\
\hline C14:1 & Methyl myristoleate & 1.24 & 1.29 & 0.056 & 0.410 \\
\hline C15:1 & Methyl cis-10-pentadecenoate & $0.27^{\mathrm{b}}$ & $0.32^{\mathrm{a}}$ & 0.009 & 0.039 \\
\hline $\mathrm{C} 16: 1$ & Palmitoleic acid & $1.43^{b}$ & $1.57^{\mathrm{a}}$ & 0.065 & 0.031 \\
\hline C17:1 & Methyl cis-10-heptadecenoate & 0.18 & 0.15 & 0.004 & 0.071 \\
\hline $\begin{array}{l}\text { C18:1 } \\
\text { trans-9 }\end{array}$ & trans-9-octadecanoic acid (elaidic acid) & $0.53^{\mathrm{a}}$ & $0.48^{\mathrm{b}}$ & 0.012 & 0.043 \\
\hline $\begin{array}{l}\text { C18:1 cis- } \\
9\end{array}$ & cis-9-octadecanoic acid (oleicacid) & 19.9 & 19.0 & 0.66 & 0.533 \\
\hline C20:1 & Cis-11-eicosenoic acid & 0.39 & 0.32 & 0.012 & 0.098 \\
\hline
\end{tabular}




\begin{tabular}{|llllll|}
\hline C22:1 & Cis-13- decosahedaenoic acid & 0.19 & 0.15 & 0.010 & 0.064 \\
\hline PUFA & & $3.65^{\mathrm{a}}$ & $3.08^{\mathrm{b}}$ & 0.096 & 0.013 \\
\hline $\begin{array}{l}\text { C18:2 } \\
\text { trans-6 }\end{array}$ & $\begin{array}{l}\text { transcis-9, 12-octadecadienoic acid } \\
\text { (translinoleic acid) }\end{array}$ & 0.086 & 0.073 & 0.0078 & 0.407 \\
\hline $\begin{array}{l}\text { C18:2 cis- } \\
6\end{array}$ & $\begin{array}{l}\text { Cis-9,12-octadecadienoic acid (linoleic acid) } \\
\text { C18:3n6 }\end{array}$ & $2.45^{\mathrm{a}}$ & $2.12^{\mathrm{b}}$ & 0.046 & 0.011 \\
\hline C18:3n3 & $\begin{array}{l}\text { Cis,cis,cis-6,9,12-octadecatrienoic acid ( } \mathrm{Y}^{-} \\
\text {linolenic acid) }\end{array}$ & 0.45 & 0.32 & 0.044 & 0.162 \\
\hline C2is,cis,cis-9,12,15-octadecatrienoic acid (a- & linolenic acid) & $0.34^{\mathrm{a}}$ & $0.29^{\mathrm{b}}$ & 0.017 & 0.031 \\
\hline C20:3n3 & Cis, cis, 11, 14-eicosadienoic acid & 0.078 & 0.073 & 0.0068 & 0.076 \\
\hline C20:4n6 & Arachidonic acid & 0.12 & 0.09 & 0.005 & 0.054 \\
\hline SMCFA & & 0.13 & 0.11 & 0.008 & 0.089 \\
\hline LCFA & & $11.9^{\mathrm{b}}$ & $14.2^{\mathrm{a}}$ & 0.24 & $<0.001$ \\
\hline
\end{tabular}

$\mathrm{C}$ = control group; I = inulin group; SFA = saturated fatty acid; UFA = unsaturated fatty acid; MUFA = monounsaturated fatty acid; PUFA = polyunsaturated fatty acids; SMCFA = short and medium-chain fatty acids; LCFA = long-chain fatty acids; SEM = standard error of mean; ${ }^{a}$ b within a row, different letters means differed significantly $(P<0.05)$.

Table 3. Effect of inulin supplementation in dietary on serum indexes

\begin{tabular}{|lllll|}
\hline Items & $\mathbf{C}(\mathbf{n}=\mathbf{8})$ & $\mathbf{I}(\mathbf{n}=\mathbf{8})$ & SEM & $P$-value \\
\hline $\mathrm{TC}(\mathrm{mmol} / \mathrm{L})$ & $8.01^{\mathrm{a}}$ & $6.49^{\mathrm{b}}$ & 0.434 & 0.008 \\
\hline $\mathrm{TG}(\mathrm{mmol} / \mathrm{L})$ & $0.479^{\mathrm{a}}$ & $0.409^{\mathrm{b}}$ & 0.0152 & 0.01 \\
\hline $\mathrm{TP}(\mathrm{g} / \mathrm{L})$ & 69.9 & 71.8 & 1.20 & 0.084 \\
\hline $\mathrm{ALB}(\mathrm{g} / \mathrm{L})$ & 34.8 & 36.6 & 0.83 & 0.086 \\
\hline $\mathrm{GLO}(\mathrm{g} / \mathrm{L})$ & 35.1 & 34.2 & 0.99 & 0.648 \\
\hline $\mathrm{BUN}(\mathrm{mmol} / \mathrm{L})$ & 5.84 & 5.32 & 0.332 & 0.051 \\
\hline
\end{tabular}

$\mathrm{C}=$ control group; $\mathrm{I}=$ inulin group $; \mathrm{TC}=$ total cholesterol; $\mathrm{TG}=$ triglyceride; $\mathrm{TP}=$ total protein; $\mathrm{ALB}=$ albumin; $\mathrm{GLO}$ = globulin; $\mathrm{BUN}=$ blood urea nitrogen; $\mathrm{SEM}=$ standard error of mean; ${ }^{\mathrm{a}, \mathrm{b}}$ within a row, different letters means differed significantly $(P<0.05)$. 
Table 4. Effect of inulin supplementation in dietary on rumen fermentation parameters

\begin{tabular}{|lllll|}
\hline Items & $\mathbf{C}(\mathbf{n}=8)$ & $\mathrm{I}(\mathbf{n}=\mathbf{8})$ & SEM & P-value \\
\hline $\mathrm{pH}$ & $6.56^{\mathrm{a}}$ & $6.30^{\mathrm{b}}$ & 0.015 & 0.040 \\
\hline Acetate $(\mathrm{mmol} / \mathrm{L})$ & $67.3^{\mathrm{b}}$ & $87.1^{\mathrm{a}}$ & 3.131 & $<0.001$ \\
\hline Propionate $(\mathrm{mmol} / \mathrm{L})$ & $25.8^{\mathrm{b}}$ & $31.1^{\mathrm{a}}$ & 0.98 & 0.003 \\
\hline $\mathrm{A} / \mathrm{P}$ & 2.61 & 2.80 & 0.08 & 0.179 \\
\hline Butyrate $(\mathrm{mmol} / \mathrm{L})$ & $11.3^{\mathrm{b}}$ & $17.5^{\mathrm{a}}$ & 0.86 & $<0.001$ \\
\hline Isobutyrate $(\mathrm{mmol} / \mathrm{L})$ & $1.05^{\mathrm{b}}$ & $1.25^{\mathrm{a}}$ & 0.037 & 0.002 \\
\hline Valetate $(\mathrm{mmol} / \mathrm{L})$ & $1.58^{\mathrm{b}}$ & $2.53^{\mathrm{a}}$ & 0.170 & 0.001 \\
\hline Isovaletate $(\mathrm{mmol} / \mathrm{L})$ & $2.08^{\mathrm{b}}$ & $2.69^{\mathrm{a}}$ & 0.101 & $<0.001$ \\
\hline $\mathrm{LA}(\mathrm{mmol} / \mathrm{L})$ & $0.71^{\mathrm{b}}$ & $0.99^{\mathrm{a}}$ & 0.027 & 0.043 \\
\hline $\mathrm{RUN}(\mathrm{mmol} / \mathrm{L})$ & 5.26 & 5.30 & 0.332 & 0.837 \\
\hline $\mathrm{NH}_{3^{-} \mathrm{N}(\mathrm{mg} / \mathrm{dL})}$ & $12.1^{\mathrm{a}}$ & $9.30^{\mathrm{b}}$ & 1.07 & 0.024 \\
\hline
\end{tabular}

$\mathrm{C}=$ control group; $\mathrm{I}$ = inulin group; $\mathrm{A} / \mathrm{P}=$ acetate to propionate ratio; $\mathrm{LA}=$ lactic acid; $\mathrm{RUN}$ = rumen urea nitrogen; SEM = standard error of mean; ${ }^{a, b}$ within a row, different letters means differed significantly $(P<$ 0.05).

Table 5. Effects of inulin supplementation in dietary on a-diversity of ruminal microbiota

\begin{tabular}{|lllll|}
\hline Items & $\mathbf{C}(\mathbf{n}=\mathbf{8})$ & $\mathbf{I}(\mathbf{n}=\mathbf{8})$ & SEM & $P$-value \\
\hline Sobs & 1446 & 1480 & 28.5 & 0.568 \\
\hline ACE & $1726^{\mathrm{b}}$ & $1801^{\mathrm{a}}$ & 27.9 & 0.031 \\
\hline Chao & $1742^{\mathrm{b}}$ & $1813^{\mathrm{a}}$ & 27.9 & 0.017 \\
\hline Shannon & $5.66^{\mathrm{b}}$ & $6.19^{\mathrm{a}}$ & 0.044 & 0.026 \\
\hline Simpson & 0.010 & 0.012 & 0.0006 & 0.071 \\
\hline Coverage & 0.98 & 0.99 & 0.001 & 0.765 \\
\hline
\end{tabular}

$\mathrm{C}=$ control group; $\mathrm{I}$ = inulin group; $\mathrm{SEM}=$ standard error of mean; ${ }^{\mathrm{a}} \mathrm{b}$ within a row, different letters means differed significantly $(P<0.05)$. 
Table 6. Differential metabolic pathway enrichment analysis of significantly differential metabolites in rumen

\begin{tabular}{|c|c|c|c|}
\hline Super pathway & Sub pathway description & Metabolites & $\begin{array}{l}P \\
\text { value }\end{array}$ \\
\hline \multicolumn{4}{|c|}{ Downregulated in inulin group } \\
\hline \multirow[t]{5}{*}{ Lipid metabolism } & Glycerophospholipid metabolism & \multirow[t]{2}{*}{ LysoPC(18:1(9Z)) } & 0.006 \\
\hline & Choline metabolism & & 0.011 \\
\hline & Glycerophospholipid metabolism & \multirow[t]{2}{*}{ LysoPC(16:0) } & 0.004 \\
\hline & Choline metabolism & & 0.014 \\
\hline & $\begin{array}{l}\text { Glycerophosphocholines } \\
\text { metabolism }\end{array}$ & LysoPC(18:2(9Z,12Z)) & 0.018 \\
\hline \multirow[t]{3}{*}{ Amino acid metabolism } & / & $\begin{array}{l}\text { Phenylmethylglycidic } \\
\text { ester }\end{array}$ & 0.018 \\
\hline & Lysine degradation & N-Acetylcadaverine & 0.013 \\
\hline & Leucine and valine pathway & 8-Methylnonenoate & 0.030 \\
\hline \multicolumn{4}{|c|}{ Upregulated in inulin group } \\
\hline Vitamin metabolism & Biotin metabolism & \multirow[t]{2}{*}{ L-Lysine } & 0.017 \\
\hline \multirow[t]{8}{*}{ Amino acid metabolism } & Lysine biosynthesis & & 0.023 \\
\hline & Arginine and proline metabolism & \multirow[t]{2}{*}{ L-Proline } & 0.006 \\
\hline & Aminoacyl-tRNA biosynthesis & & 0.013 \\
\hline & Phenylalanine metabolism & \multirow[t]{3}{*}{ L-Phenylalanine } & 0.025 \\
\hline & $\begin{array}{l}\text { Phenylalanine, tyrosine and } \\
\text { tryptophan biosynthesis }\end{array}$ & & 0.036 \\
\hline & Phenylpropanoid biosynthesis & & 0.017 \\
\hline & Tyrosine metabolism & \multirow[t]{2}{*}{ L-Tyrosine } & 0.001 \\
\hline & Biosynthesis of shikimate pathway & & 0.013 \\
\hline Nucleotide metabolism & Pyrimidine metabolism & \multirow[t]{2}{*}{ Uracil } & 0.008 \\
\hline Vitamin metabolism & $\begin{array}{l}\text { Pantothenate and CoA } \\
\text { biosynthesis }\end{array}$ & & 0.006 \\
\hline \multirow{2}{*}{$\begin{array}{l}\text { Biosynthesis of plant } \\
\text { secondary metabolites }\end{array}$} & Steroid synthesis & Deltonin & 0.034 \\
\hline & Isoflavonoid biosynthesis & Daidzein & 0.029 \\
\hline
\end{tabular}


/ = the metabolic pathway was not annotated.

\section{Figures}

$\mathbf{A}$

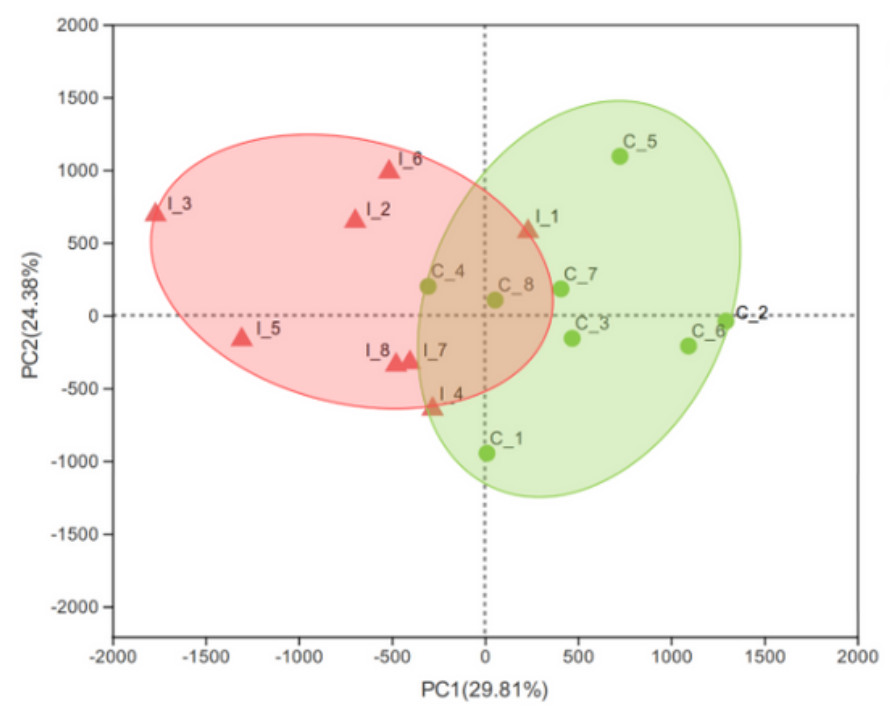

B

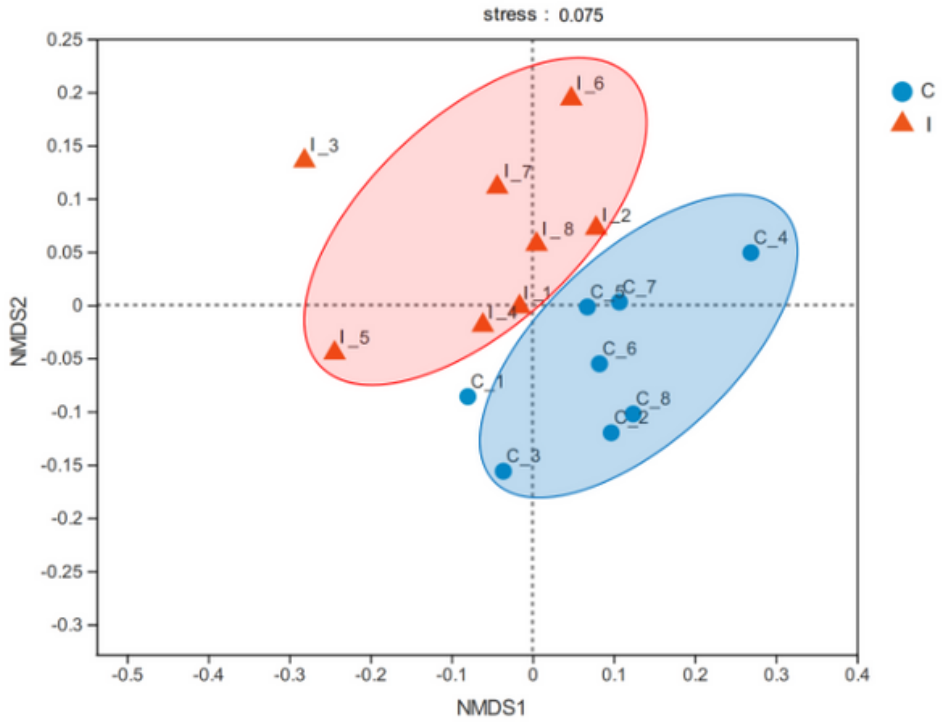

Figure 1

Beta diversity analysis of ruminal microbiota through (A) principal coordinate analysis (PCoA) and (B) Non-metric multidimensional scaling analysis (NMDS). C, control group. I, inulin group. 
A
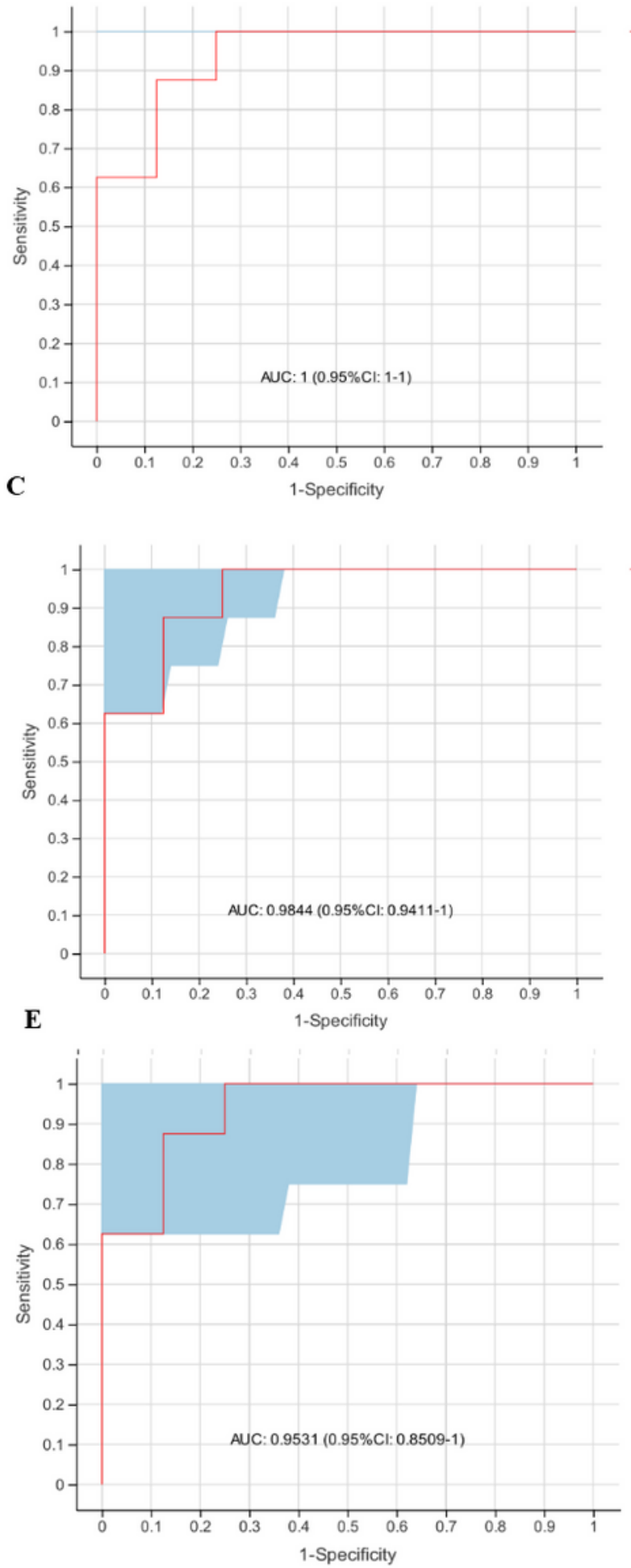

B

D
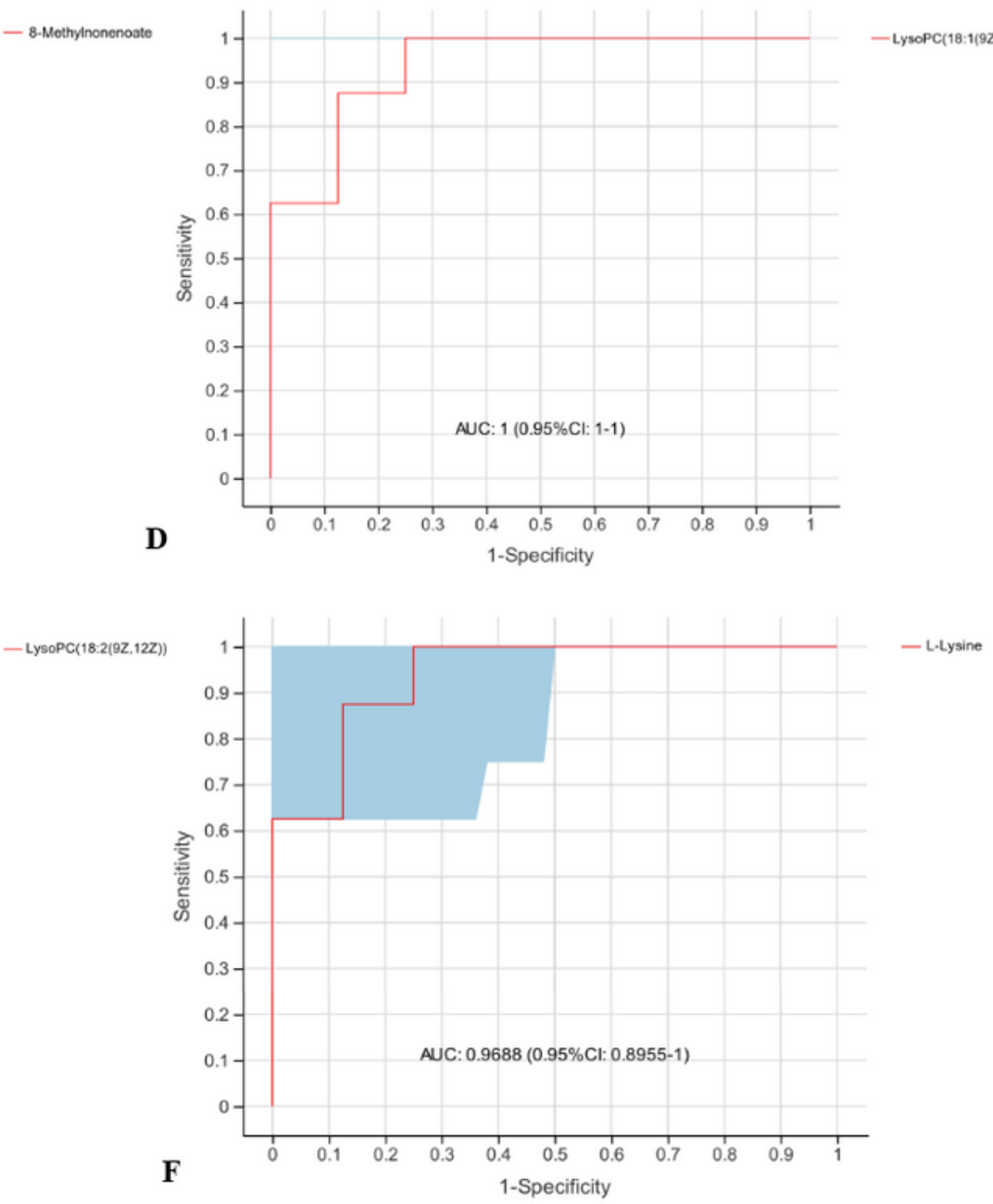

- LysoPC(16:0)

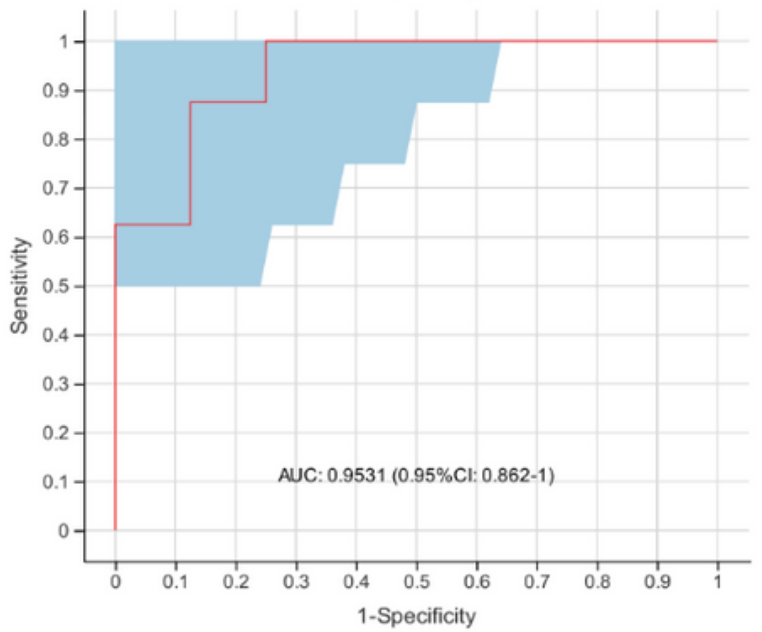

\section{Figure 1}

Receiver operating characteristic (ROC) curves to evaluate the differential metabolites that have key impact on the differentiation between the two groups. ROC curve reflects the relationship between sensitivity and specificity. The $x$-axis is specificity (false positive rate). The closer the $x$-axis is to zero, the higher the accuracy will be. The y-axis is sensitivity (true positive rate). The larger the y-axis is, the better the accuracy is. The Area under curve (AUC) is used to indicate the accuracy of prediction. AUC value 
close to 1 suggested the higher the accuracy of prediction. (A) 8-Methylnonenoate; (B) LysoPC (18:1(9Z)); (C) LysoPC (18:2(9Z,12Z)); (D) L-Lysine; (E) LysoPC (16:0); (F) L-Proline.
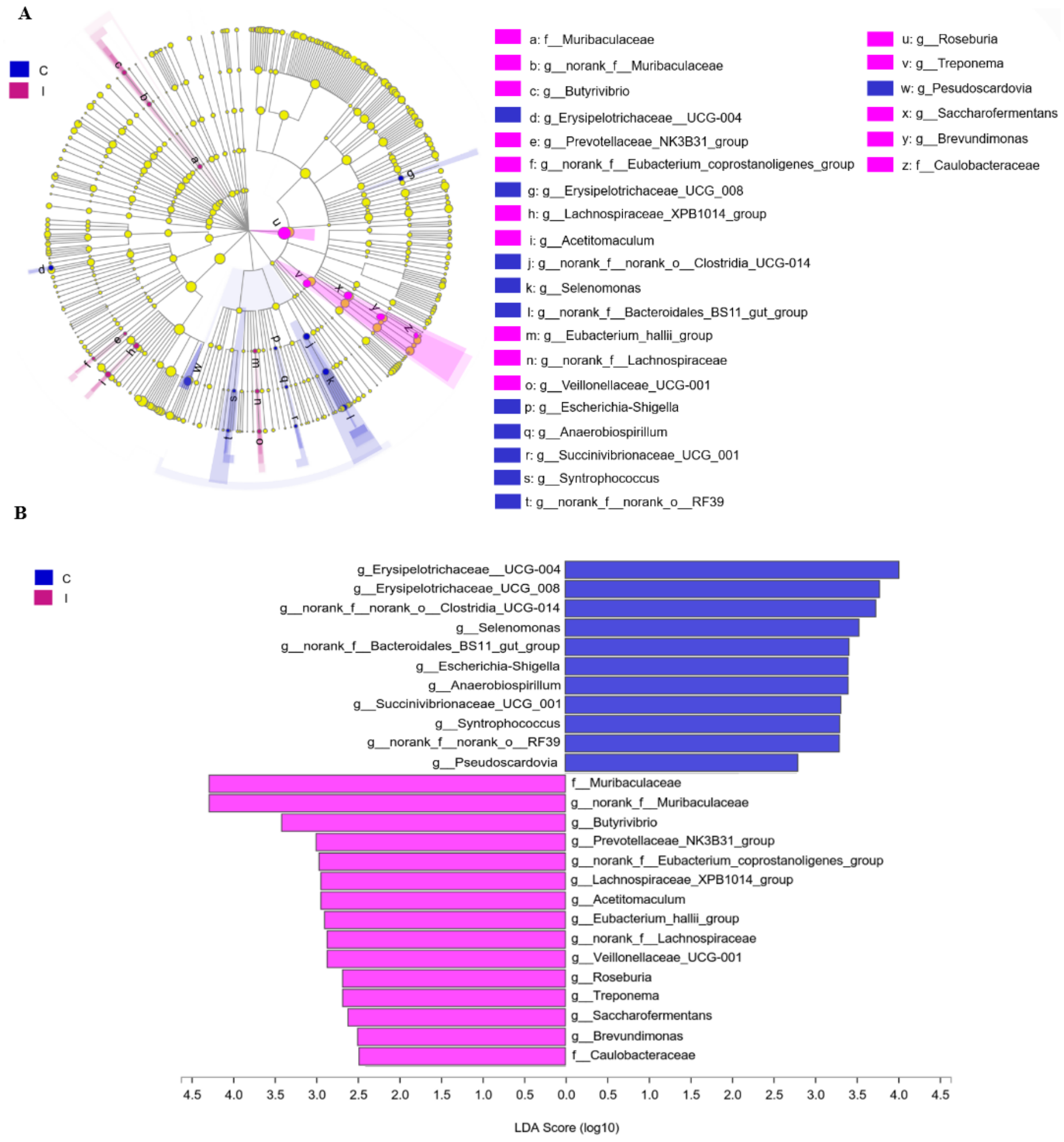

\section{Figure 1}

The linear discriminant analysis effect size (LEfSe) analysis of differential ruminal microorganisms in control and inulin group. (A) Cladogram showed the significantly different bacteria from phylum to genus level. The nodes with different color represent the microbes that are significantly enriched in the 
corresponding groups and have a significant influence on the difference between the two groups. The yellow nodes represent the microbes that have no significant difference between the two groups. (B) Linear discriminant analysis (LDA) bar showed the impact of the abundance of each species on the difference between the two groups. P-value $>0.05$ and LDA score $>2.5$ were defined as significant difference.
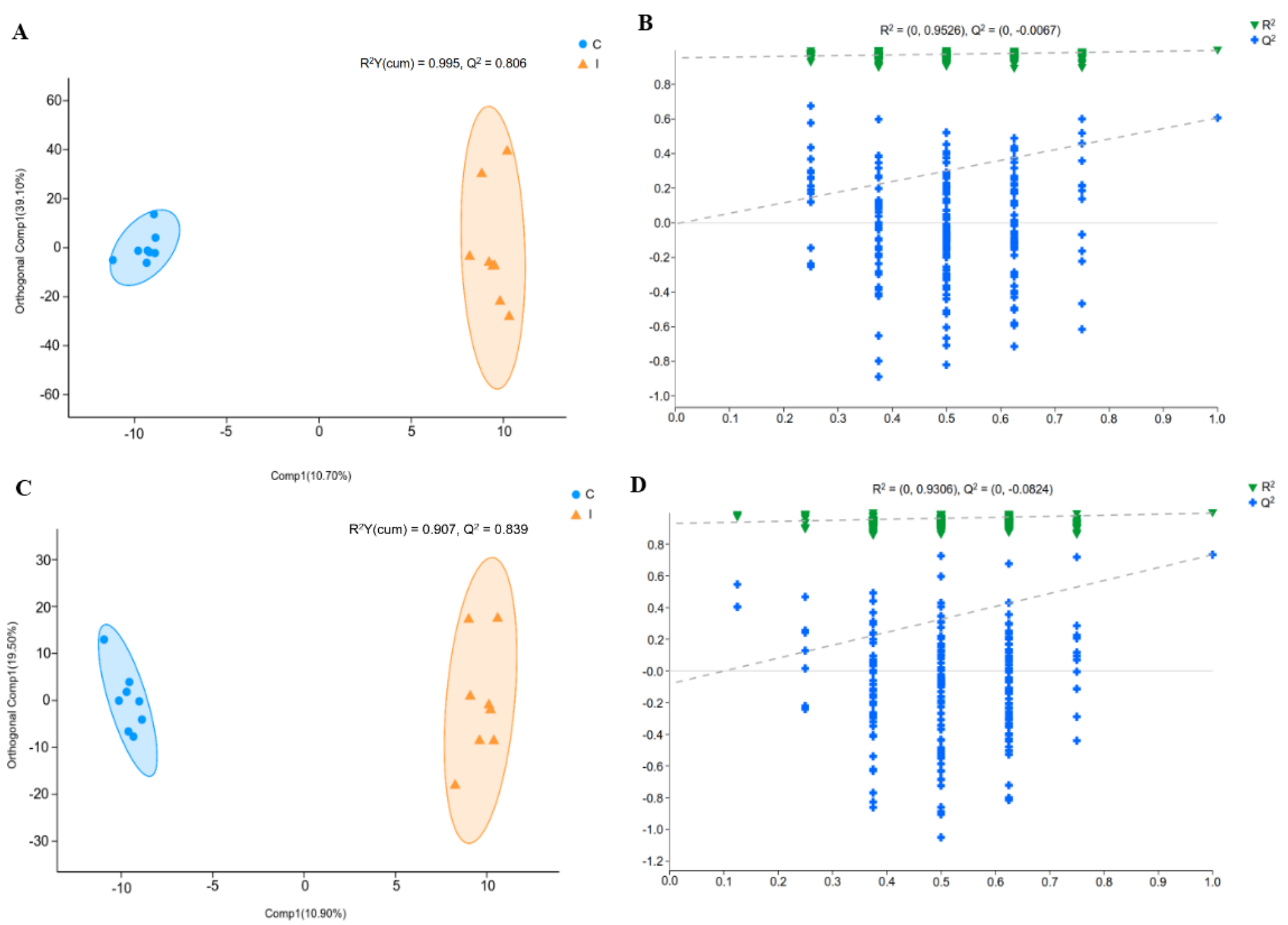

Figure 1

Orthogonal partial least squares discriminant analysis (OPLS-DA) plot (A, $C$ ) and response permutation testing (RPT) (B, D) of rumen metabolites in comparisons of the control and inulin groups following $(A, B)$ positive and $(C, D)$ negative mode ionization. R2Y (cum) and Q2 indicates the cumulative interpretation power and predictive power of the model, respectively. C, control group; I, inulin group. 
A

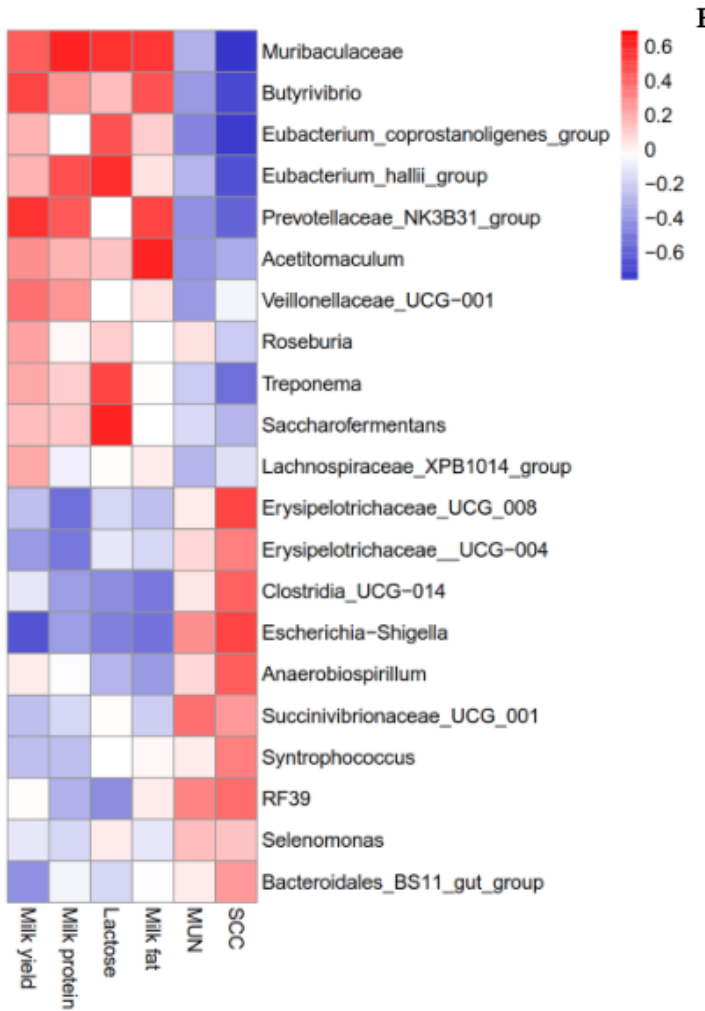

B

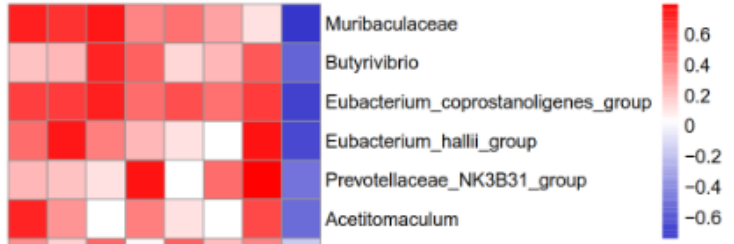

Veillonellaceae_UCG-001

Roseburia

Treponema

Saccharofermentans

Lachnospiraceae_XPB1014_group

Erysipelotrichaceae_UCG_008

Erysipelotrichaceae_UCG-004

Clostridia_UCG-014

Escherichia-Shigella

Anaerobiospirillum

Succinivibrionaceae_UCG_001

Syntrophococcus

RF39

Selenomonas

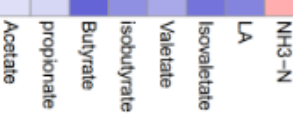

C

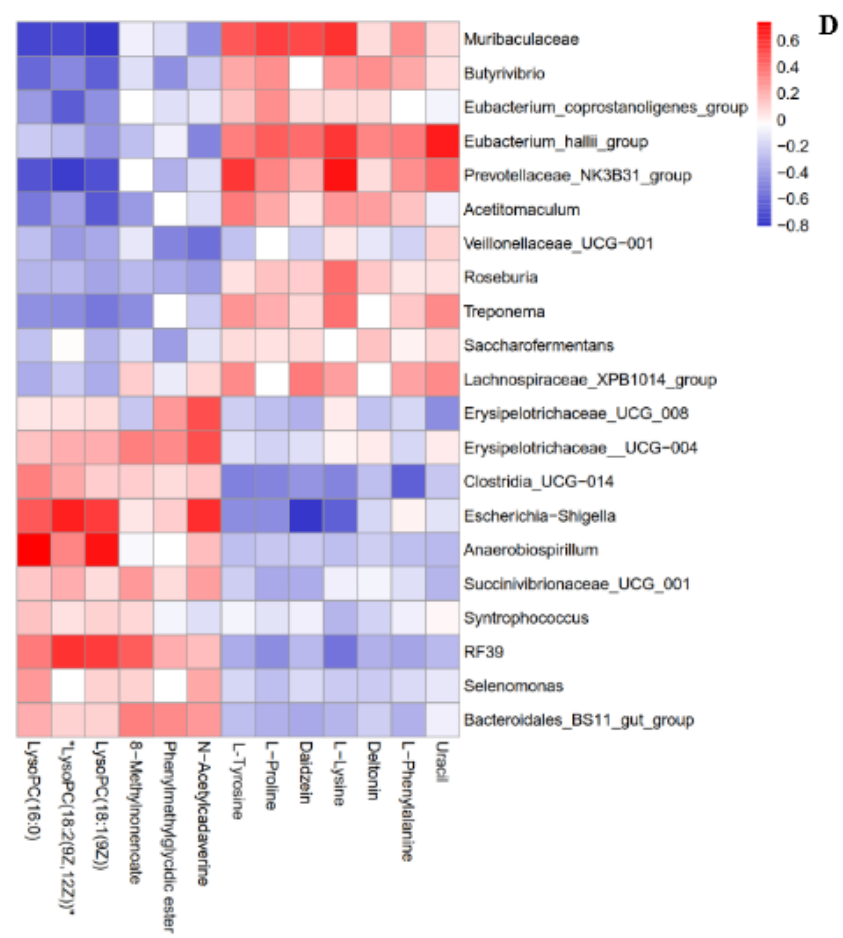

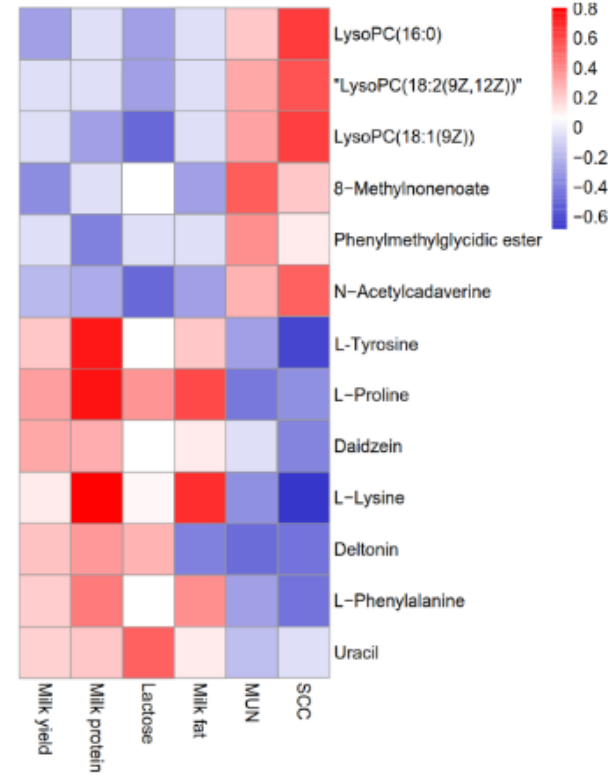

\section{Figure 1}

Correlation analysis between (A) significantly differential bacteria and milk components, (B) significantly differential bacteria and rumen fermentation parameters, (C) significantly differential bacteria and differential metabolites, as well as (D) significantly differential metabolites and milk compositions. LA, lactic acid; SCC, somatic cell counts; MUN, milk urea nitrogen. Red indicates a positive correlation, while the blue indicates a negative correlation. 
A

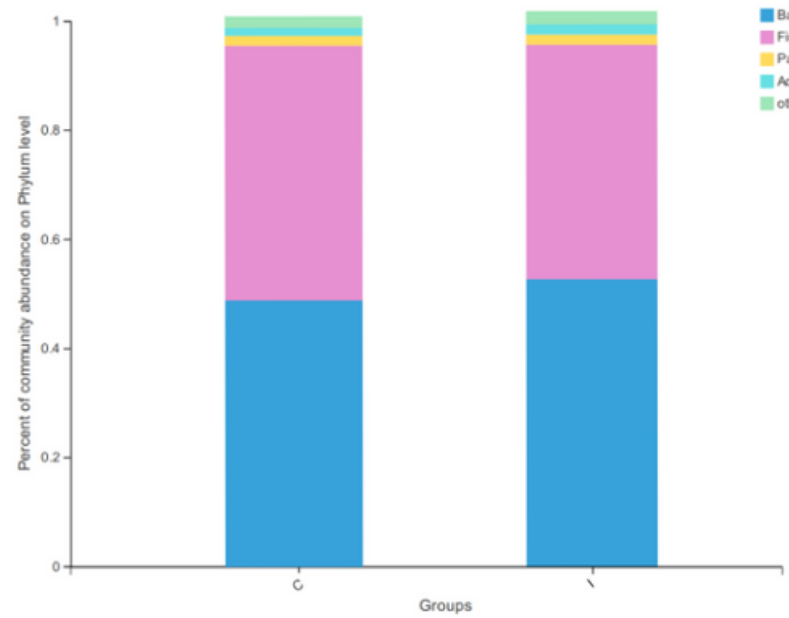

B

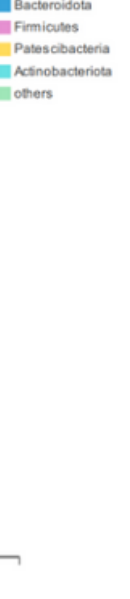

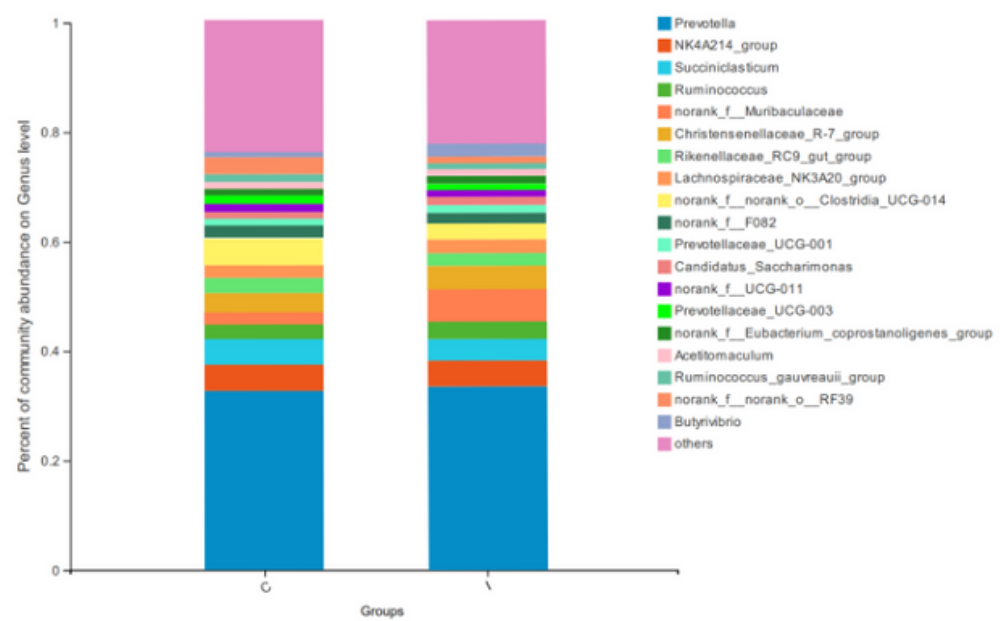

Figure 1

The ruminal bacterial community compositions in control and inulin groups at (A) phylum and (B) genus level. C, control group. I, inulin group. The different colors of the bars represent different species, and the length of the bars represents the proportion of the species.

\section{Supplementary Files}

This is a list of supplementary files associated with this preprint. Click to download.

- Additionalfile1SupplementaryTables.docx

- Additionalfile1SupplementaryFigures.docx 